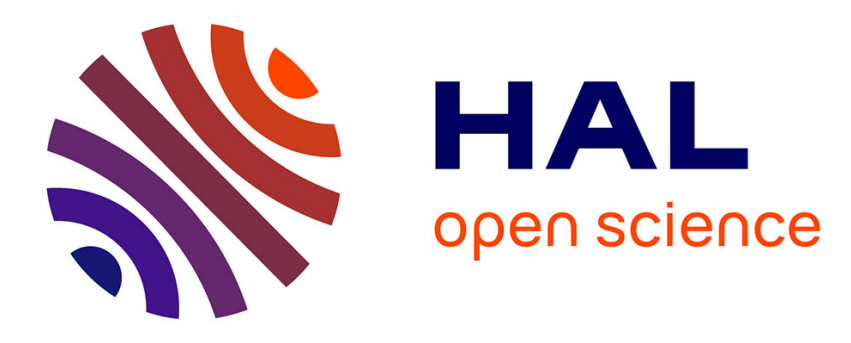

\title{
Les textes techniques de l'Antiquité. Sources, études et perspectives
}

Philippe Fleury

\section{To cite this version:}

Philippe Fleury. Les textes techniques de l'Antiquité. Sources, études et perspectives. Euphrosyne. Revista de filologia clássica, 1990, 18, pp.359-394. hal-01609488

\section{HAL Id: hal-01609488 \\ https://hal.science/hal-01609488}

Submitted on 3 Oct 2017

HAL is a multi-disciplinary open access archive for the deposit and dissemination of scientific research documents, whether they are published or not. The documents may come from teaching and research institutions in France or abroad, or from public or private research centers.
L'archive ouverte pluridisciplinaire HAL, est destinée au dépôt et à la diffusion de documents scientifiques de niveau recherche, publiés ou non, émanant des établissements d'enseignement et de recherche français ou étrangers, des laboratoires publics ou privés. 


\section{EVPHROSYNE}

REVISTA DE FILOLOGIA CLÁSSICA

NOVA SÉRIE

VOLUME XVIII

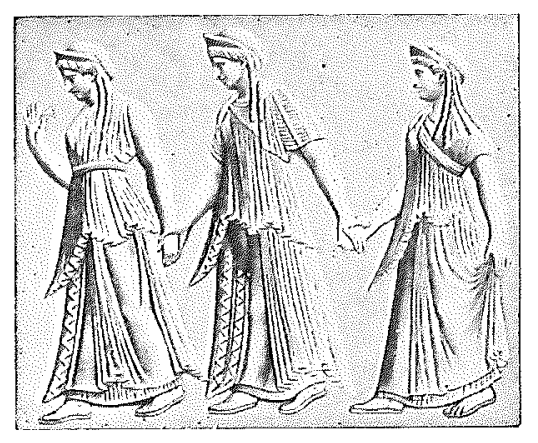

INSTITUTO NACIONAL DE INVESTIGAÇÃO CIENTÍFICA

CENTRO DE ESTUDOS CLÁSSICOS

FACULDADE DE LETRAS DE LISBOA 
Les textes techniques de l'Antiquité

Sources, études et perspectives

Lorsque l'adjectif «technique» est employé avec le mot «texte» il prend des acceptions diverses suivant les locuteurs ou quelquefois, pour un mêne locuteur, suivant le conterte. Aifférts d'emploi d'une machine, l'exposé d'une découverte technodifférents que le mode d'énés teichniques, l'analyse d'un fait médical ou encore l'histoire d'un phénomène grammatical. En réalité «texte technique» s'entend aussı bien pour «texte spécialisé» que pour «texte sur une technique». Le mot reçoit le premier sens dans une étude qui reste fondamentale pour nos disciplines: "La litté. rature technique des Grecs et des Latins" [1] ${ }^{1}$, puisque J. Beaujeu applique l'expression «littérature technique» à toute la littérature scientifique et technique et qu'il traite en même temps des sciences exactes, des sciences de la nature, des sciences occultes, de la mécecine, des techulure) propres disciplines littéraires (commentateurs, grammairiens, érudits, textes juridiques). Pour notre part, nous prendrons ici le mot dans sa seconde acception et nous traiterons des textes sur les techniques. Reste à définir toutefois ce que nous entendons par "technique». Si une définition telle que «ensemble des procécés d'un art ou d'un métier, paraît claire à première vue, elle n'est pas si facile à utiliser comme critère de classement pour les textes antiques. D'abord l'émergence de la distinction entre science et technique dans la terminologie a eté progressive ; ensuite les rapports

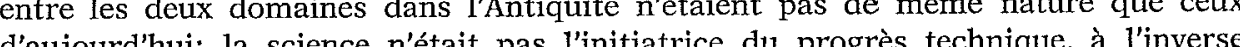

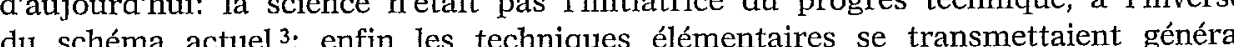
lement de bouche à oreille, sans support écrit. Sans aller aussi loin que M. Daumas qui nie implicitement l'existence d'une littérature technique avant le XVIII siècle: «il y a un peu plus de deux siècles seulement que les connaissances techniques se transmettent autrement que par le geste et la parole» ${ }^{4}$, il est vrai qu'en dehor

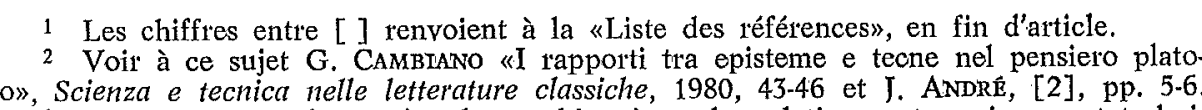
nicos, 3 On trouvera un bon point de vue historique des relations entre sciences et techni ques dans la preface
pp. V-XVI. 
de quelques inscriptions nous ne possédons presque aucun texte destiné directement à un technien proprement dit, c'est-à-dire à un homme de métier, si ce n'est peutte-etre les recueils de recettes ${ }^{5}$ (sidérurgiques ou autres) et les traités des arpenteurs. Nous avons traité ailleurs ${ }^{6}$ la question des destinataires des ouvrages que explicites qui sont le pluis ils ils s'adressent presque tous, outre leurs destinataires public cultivé, pas nécessairement technicien. Du reste, ou le chef de l'Etat, a un J. J. Hall [4], les difficultés matérielles de la publication des textes dans l'Antiquité ont probablement été une gêne dans l'avancée du progrès technique. Quoi qu'il en soit, il reste une littérature grecque et romaine qui traite directement ou indirectement des techniques et ce sont ces textes que nous voudrions essayer de cerner ici.

Toute étude sur les textes antiques doit d'abord se pencher sur les problèmes de méthode et sur la nature des objets observés: un texte de Sénèque sur l'origine des vents el un texte de Caton sur lattitude du proprietaire face a son fermier ne uspécialiséen même nature, bien qu'appartenant tous deux a la littérature dite parfaitement, il faut faire appel à des références différentes pour les comprendre qu'il y ait cloisonnement entre les oeures: le De rerum es. Cela ne signifie pas l'on n'hésite pas à ranger parmi la littérature scientifique, a influencé deséce, que techniques aussi différentes que les Georgica de Virgile ou le De architectura de Vitruve 7 . Mais la notion de "famille de texte" nous paraît importante car elle correspond à une réalité antique. Il existe par exemple une famille regroupant les auteurs sur la construction, la mécanique et la tactique militaire. Certains de ces auteurs ont écrit dans les trois domaines à la fois: l'oeuvre connue de Philon de Byzance 8 en est une belle illustration et tout un tissu de relation fortification. Quand Vitruve entreprend de, de mécanique, de stratagèmes ou de regroupe ces domaines, de facon inérale il est vrai, mais lo De architectura trite de la construction civile et militaire, de la mécanique civile et militaire et comporte quelques chapitres qui appartiennent au genre des «stratagèmes" 9 . Il apparât qu'une etude portant sur ces textes aura un caractere homogene et, en y ajoutant le domaine de l'agriculture, nous couvrons ainsi l'ensemble des techniques de base nécessaires à la vie humaine: la nourriture, la construction et la guerre. La médecine occupe déja une place un peu particulière: mal dégagée des pratiques médicale (notamment par l'acte chi elle est à la fois technique par la pratique physiologiques. Nous aurions pu trater ici une partie des textes du copus mélical

3 Voir à se sujet la bonne introduction de R, Halzeux au premier tome de son
édition des alchimistes grecs [15], pp. 24-30

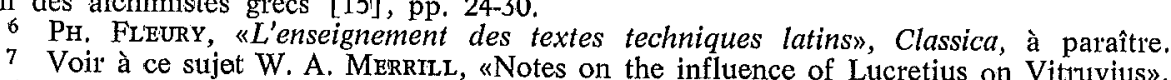
$\begin{aligned} & 7 \text { Voir à ce sujet W. A. } \\ & \text { TAPhA } 35,1904, \text { p. XVI.XXI }\end{aligned}$

nous voyons additionnant les cuvres conservées et celles que nous connaissons par mentions, tiques, automates, levage et traction ...), la gnomonique, l'architecture civile (ports) et militaire, la mécanique militaire, les stratagèmes (messages secrets).
9 Arch. 10, 16, (notamment Oreibasios ${ }^{10}$ ), mais, dans la mesure où ils partie d'une domaine bien défini et abondamment étudié par ailleutrs, nous ne les avons pas retenus. Quan a pose des problèmes différents.

Dans l'inventaire des sources techniques, nous nous méfierons des classifications habituelles car elles ne rendent pas toujours compte de la spécificité de ce type de texte. Par exemple le De bello gallico ou le De bello ciuili de César, les Res gestae d'Ammien Marcelin ne sont pas ordinairement classés parmi les oeuvres techniques. Pourtant Gall. 4,17 (le pont sur le Rhin), Ciu. 2, 2-16 (le siège de Marseille), Amm. 23,4 (description des machines de siège) sont des textes

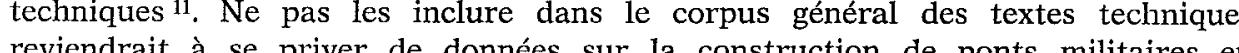
campagne ou sur l'évolution de l'artillerie et à ignorer toute une partie de littérature technique qui se trouve insérée dans des oeuvres d'un autre caractère. L'inventaire qui va suivre tient compte de ces considérations et nous entendons par «texte technique» aussi bien l'oeuvre complète d'un auteur qu'une de ses oeuvres ou une partie d'une de ses oeuvres. Pour reprendre une classification anteressante proposee par J. Gallard et R. Martin dans leur introduction aux Genres litteraires à Rome ${ }^{12}$, nous nous situons dans le "genre démonstratif" et didtextes auxquels nous faisons no poésie limitons à l'exposé de techniques, c'est-à-dire d'uactivités ce genre, nous nous pour objet de recueillir, d'adapter et 'de transformer les matériaux naturels of d'améliorer les conditions de son existence» 13 , activités auxquelles nous ajouton la conduite des opérations militaires tellement celles-ci sont partie intégrante de vie quotidienne dans l'Antiguité et appartiennent à la même «famille» de préoccupations que l'architecture ou la mécanique.

Nous allons d'abord proposer un inventaire des textes techniques de l'Antiquité en distinguant les recueils, puis la littérature grecque et la littérature latine. c'est une des caractéristiques de ce type de littérature, les auteurs participa souvent aux diverses branches de la famille technique. Un classement thématiquent est toutefois proposé en annexe. Nous ne tenons compte que des textes d'une certaine etendue et, bien que la frontière ne soit pas toujours facile à tracer entre fragment et texte complet 15 , nous pensons que la littérature fragmentaire pourrait

10 Voir par exemple la traduction de quelques extraits techniques dans A. G. DrACH MANN 11 Les philologues se sont du reste parfois demandé si les deux premiers n'avaient
pas pour auteur un ingénieur de César.

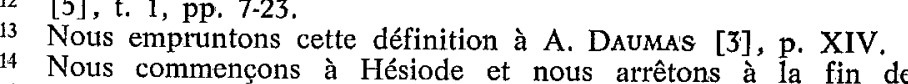

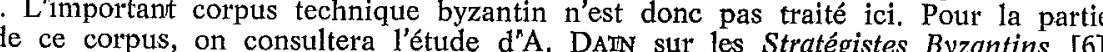
qui, malgré son titre, ne se limite pas a la période byzantine, mais recense tous les textes
des stratégistes grecs, depuis Enée le Tacticien jusqu'au XV' siècle $p$. C.: pour les travaur antếrieurs à 1966 , elle fournit une bibliographie commode.

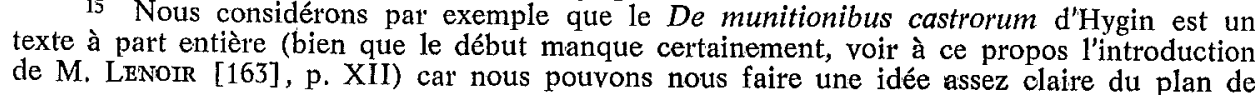


faire l'objet d'un recensement qui lui serait propre en tenant compte de certaines particularités, notamment la difficulté de définir le caractère d'une oeuvre dont

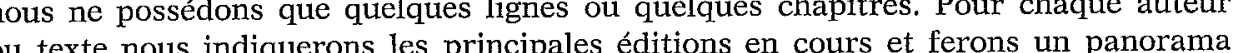
des études qui leur ont été consacrées ces vingt dernières années. Nous ne donnons des références antérieures à 1969 que pour des textes peu étudiés ou dont la bibliographie est difficile à rassembler et, dans ce cas, seulement pour des éditions ou des études qui n'ont pas été remplacées ${ }^{16}$. Nous ne tenons pas compte des articles des dictionnaires (IOxford Classical Dictionary dans sa seconde édition de 1970 par exemple) ou des grandes encyclopédies comme la Realency. clopäalie der klassischen Alterninswissensohnjt de Pauly et Wissowa car les rubriques cos deripes textes techniques, puis sur leur contenu.

Les textes techniques grecs et latins n'ont jamais été réunis tous ensemble dans un même corpus. Il est vrai que celui-ci occuperait un volume considérable (nous avons relevé ci-dessous une dizaine d'auteurs grecs et une quinzaine d'auteurs latins susceptibles d'entrer dans un tel recueli, sans compter les fragments) et qu'il poserait des problèmes de définition difficilement solubles. A date ancienne cependant il était courant de réunir dans une même édition plusieurs auteurs appartenant au même domaine technique 1 . Cela s'est fait par exemple pour les Schneider [8] à partir de 1794, ou encore avec le volume de la collection Nisard 18 regroupant Caton, Varron, Columelle et Palladius, Pour les «mathématiciens, l'édition de M. Thévenot [9] en 1693 regroupe le Traité des machines d'Athénée le Mécanicien, les Poliorcétiques d'Apollodore de Damas, les livres IV et V de la Syntaxe mécanique de Philon de Byzance, le traité de Biton, les Belopoiica, la Chirobaltste, les Pheumatiques, les Automates de Heron d Aelxandrie, les Cestes de Jules l'Africain et divers autres opuscules ou fragments. Le texte, fondé sur un seul manuscrit du XVIs siecle, est aujourd hui largenent dépassé, mais il sert

l'ouvrage, mais nous rejetons parmi les fragments le De hortis de Gargilius Martialis.
Pourtant ce dernier a fait l'objet d'une édition séparée en 1978 par I. MAzzINI (Bologne, Patron). Nous faisons une exception avec quelques éditions de textes classiques pou
16. 16 Nous faisons une exception avec quelques éditions de textes classiques pour
lesquels in peut être utile de disposer d'un choix de reférences plus vaste, et avec des types
de recueils qui n'ont pas été. remplacés et qu'il est intéressant de connaître, ant moins par de recuél.
curiosité. 17 . Cette pratique remonte en fait à l'époque de la tradition manuscrite: un manuscrit
de la bibliothèque Saint-Marc à Florence, aujourd'hui perdu, contenait à l'origine le De agride la bibliothèque Saint-Marc à Florence, aujourd'hui perdu, contenait à l'origine le De agri-
culturta de Caton, les Res rusticae de Varron, les douze livres de Columelle et Gargilius

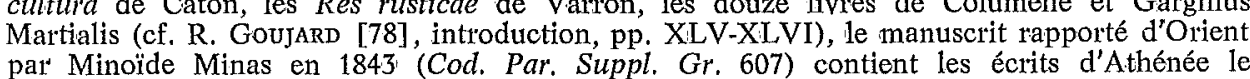

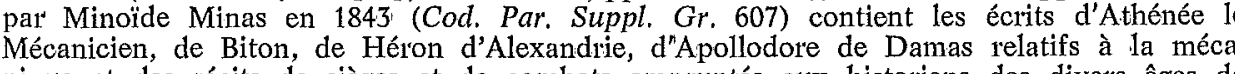
neque et des récits de sièges et de combats empruntés aux historiens des divers âges de
la Grèce depuis le siècle de Thucydide jusqu'aux premières années du Bas-Empire (cf. C. WEschrR [11], pp. X-XL). Nous ne donnons ci-dessous que quelques exemples der ereceilis anciens, on en trouvera d'autres dans les instruments bil
Engelmann-Preuss, Fabricius, Klussmann, Marouzeat ...) dits nous avons les recueils de $\mathrm{H}$. Köchly et W. Rüstow [10] en 1853, de C. Wescher [11] en $1867^{10}$ et de R. Schneider [12] à partir de $1908{ }^{20}$. Il n'existe à notre celui d'A. G Due deux recueils modes sur la méconique civile et celui d'B. W. Marsden [14] en 1971 sur l'artillerie A. G Drachman rassemble des extraits des Questions de Mécanique du Pseudo-Aristote ${ }^{21}$, de la Dioptre d'Héron 22, des Mécaniques d'Héron ${ }^{23}$, d'Oreibasios ${ }^{24}$, de Pappos ${ }^{25}$ et de Vitruve ${ }^{26}$, mais il ne propose, outre le commentaire, que la traduction anglaise sans édition du texte original. Le recueil d'E. W. Marsden est, de ce point de vue, beaucoup plus utilisable, 11 ne se repose pas sur une nouvelle étude des manuscrits: il a été préparé à partir des editions et commentaires de $\mathrm{H}$. Köchly, $\mathrm{C}$. Wescher, R. Schneider, R. Schone, H. Diels, A. Relm ou E. Schramm mais il fournit le texte, des elements ouvrage sur le développement historique de l'artillerie [259] qui avait précédé de deux ans l'édition des textes. Le recueil contient les Belopoiica de Héron, le traité de Biton, les Belopoiica de Philon, les chapitres de Vitruve sur l'artillerie ${ }^{27}$ la Chirobaliste de Héron, un court paragraphe de Végèce sur la baliste ${ }^{28}$, le passage d'Ammien ${ }^{29}$ sur la grande baliste, les chapitres du De Rebus bellicis sur Ia ballista quadrirotis et la ballista falminalis ${ }^{30}$, et un extrait de Procope sur un petit lanceur. A ces recueils de textes il serait possible d'ajouter les recueils de fragments des gromatici ueteres ${ }^{32}$, des metrologici scriptores ${ }^{33}$ ou encore des des Universités de France sous la direction d' $\mathrm{H}$. D. Saffrey ${ }^{34} \mathrm{R}$. Halleux [15] 'auteur du premier des douze volumes prévus 35 qui traite de la purification de

19 C. Wescher utilise pour la première fois le manuscrit de Minas (IX'e ou $\mathrm{X}^{\mathrm{e}}$ siècle) Chirobaliste), Apollodore de Damas et de l'Anonyme de Bologne. Cette édition sert géné. ralement de base aux références encore utilisées aujourd'hui.
20 Ce recueil contient les Poliorcétiques d'Apollodore de Damas (avec index et dessins des manuscrits), 1 'Anonyme de Byzance (avec index et dessins), le Traité des des machines
d'A Anthén

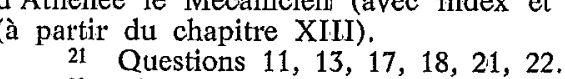

23
24
2 Livre 1, chap. 1, 15, 18-23, 34; livre 2, chap. 1-32, 34; livre 3, chap. 1-21

25 Pp. 1060, 1110, 1114, 1118, 1120, 1122, 1126, 1128, 1130 et 1132 de l'edition

F. Huts HeH, 1878.
27 Livre IX, chap. 8; livre X, chap. 1-2, 4-9.
27 10, 10-12.

${ }_{28}^{27} 10,10-12$

29 Anm. 23, $4,1-3$.
30 De reb. bell. 7 et 18 .

31 Goth. 1, 21, 14 -18.

32 Ed. LaCHMANN et MoMmsen, 1848

33 Metrologicorum scriptorum reliquiace, éd. F. Hurtsch en deux volumes: I. Scriptores
graeci (1864), II. Scriptores romani et indices (1866), Leipzig, Teubner, réimprimé en 1971 en un seul volume.
34 Nous devons à J. M. Mathieu, Professeur à l'Université de Caen, de nous avoir

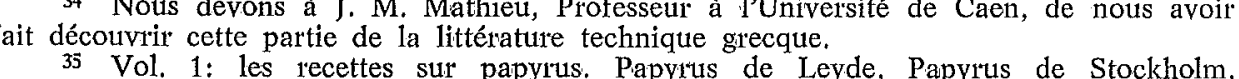
35 Vol. 1: les recettes sur papyrus. Papyytus de Leyde, Papyrus de Stockholm,
fragments der recettes. Vol 2: les vieux auteurs I (lesphysica th mystica du Ps. Démocrite.
Ostanès, Cléopâtre. Comarios. Isis à Horus. Hermes Trismegiste). Vol. 3: les vieux auteutrs II 
l'étain, du plomb, de la fabrication de l'asèm, de la teinture de l'or, du blanchiment du cuivre, du mordançage des pierres etc.... D'un point de vue général cette littérature fragmentaire des recettes est intêressante a étudier parallèlement aux autres textes ${ }^{36}$ car elle nous donne des techniques de transformation (peu importe an sagisse our anon des manuscrits Oussi bien grecs que latins. Avant de passer à l'étude individuelle des textes, il reste ì mentionner les recueils de tacticiens tel celui réalisé par l'Illinois Greek Club pour la Collection Loeb en 1948 et qui contient notamment les textes d'Enée le Tacticien, d'Asclépiodote et d'Onasandre.

\section{LITTÉRATURE GRECQUE}

HESIODE (milieu VIII ${ }^{\text {s }}$ s. a.C.). Nous rangeons parmi les textes techniques Les travaux et les jours, v. 383-617 (sur l'agriculture), v. 618-694 (sur la navigation commerciale), v. $765-828$ (sur les jours). L'oeuvre d'Hésiode est suffisamment connue et étudiée pour qu'il soit inutile de s'y étendre. Peu importe également pour notre propos si la partie didactique du poème etail a lorigine une oeuvre

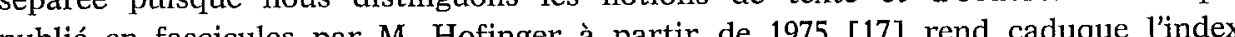

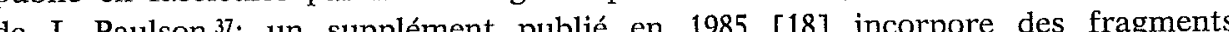

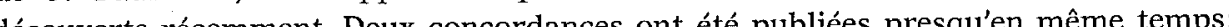
celle de W. W. Minton en 1976 [19] et celle de J. R. Tebben en 1977 [20]. Toutes les grandes collections possèdent leur édition; citons celles de R. Rzach pour Teubner en 1913 [21], de P. Mazon pour la C.U.F. en 1928 [22], de F. Solmsen pour la Scriptorum Classicorum Bibliotheca d'Oxford en 1970 [23]. En 1978 a été publiée une bonne édition commentée par M. L. West [24] avec une bibliographie sélective à la fin de l'introduction. Citons également une traduction partielle avec notes des v. 387-447 par J. Mendes de Castro en 1982 [25]. Pour une étude sur la langue, voir louvrage de G. P. Edwards en 1971 [26]; pour Thistoire de la technique à travers Hésiode, voir M. Erren [27], sur les dessins techniques des manuscrits: G. Derenzini [28]. Plusieurs chercheurs se sont intéressés ces dernières années aux aspects techniques des Travaux et des jours: L. Deroy [29] pour l'araire, J. Fuzier et P. Ghiron-Bistagne [30] pour le battage du blé, F. Buranelli [31] pour
l'outillage du travail sur le bois, N. J. Richardson, S. Piggott [32] et G. Raepsaet [33]

(la chimie de Moise. Le travail des quatre éléments. Les huit tombeaux). Vol. 4: Zosime. (Etienne d'Alexandrie). Vol. 7: les Commentateurs III (Le Chrétien, L'anonyme, Cosmas, La pierre des philosophes, Jean 1'Archiphêtrte). Vol. 8: les poèmes alchimiqueses (Heliodoros,
Theophrastos, Hierotheos, Archelaos). Vol. 9: les traités opératoires I. Vol. 10: les traités Theophrastos, Hierotheos, Archelaos). Vol. 9: les traités opératioires I. Vol. 10: les traités
opérataires II. Vol. 11: les, lexiques anciens. Vol. 12: Index genéral.

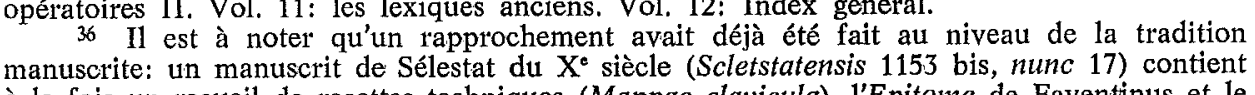
à la fois un recueil de recettes techniques (Mappae clavicho), ${ }^{\prime}$ Epitome de Favent

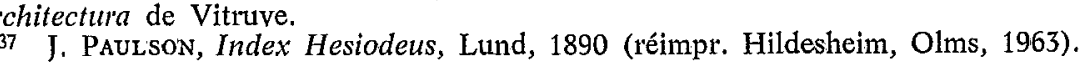

pour le chariot, G. Aujac [34] pour le calendrier agricole. Les fragments conservés d'Hesiode nous indiquent quil s'etait interesse a dautres domaines scientifiques et techniques

ENEe LE TACTICIEN (milieu IV s. a. C.). La Poliorcétique d'Enée se range davantage parmi les traites des tacticiens et serait à étudier avec les oeuvres d'ASCLEPIODOTE LE PHILOSOPHE (I I ${ }^{\circ}$ s. a.c.), d'ONASANDRE ( ${ }^{\text {ser }}$ s. a.C.), d'ELIEN (déb. du II's. p. . .), d'ARRIEN (II's. p.C.) auxquelles ou pourrait ajouter quelques passages de Xenophon et de Polybe. Mais certains chapites donnent des renseignements précis sur les machines de siège (engins d'attaque ou de défense) et, à ce titre, se rangent parni les tété remplacé à notre connaissance. Outre l'édition des tacticiens grecs dans la collection Loeb que nous avons déjà mentionnée supra nous disposons de l'édition d'A. Dain et A. M. Bon dans la C.U.F. en 1967 [36]. Trois études récentes: celles de M. Bettalli [37] et de R. Urban [38] en 1986 portent sur des questions de tactique, celle de Ph. Pattenden [39] en 1987

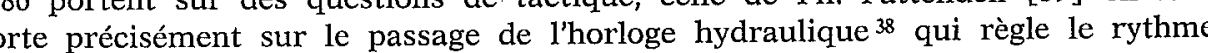
des gardes.

PSEUDO-ARISTOTE (déb. du III' s. a.C. ?). Les Questions de mécanique figurent depuis longtemps dans le corpus aristotélicien bien qu'il soit généralement figurent depuis longtemps dans le corpus aristotelicien bien quili soit genéralement II ne s'agit pas d'une oeuvre technique à proprement parler. La forme adoptée, celle des questions, la range plutôt du côté des oeuvres à vocation théorique, mais elle contient nombre de renseignements pratiques sur le fonctionnement des machines simples: poulie, treuil, levier, balance ... Ce texte est peu connu et peu etudié. La collection Loeb possède une edition qui date de 1936 [40] par W. S. Hett mais qui repose sur le texte de Bekker et Apelt en 1888 . Nous avons signale supra la traduction partocer reprise après le dócis de ce dernier. A part le commentaire d'A. G. Drachmann et la petite notice que lui consacre K. D. White [226] dans sa synthèse de 1984 (p. 177) il n'existe pas d'études récentes. On pourra utiliser l'article de M. Gallian [41] en 1901, ou consulter le livre de L. Sprague. de Camp [42] (1960), p. 118 sqq.

BITON (III ${ }^{e}$ ou II' s. a.C. ?). Son traité sur la Construction des machines de guerre et des catapultes a peu intéressé les éditeurs. 1 est vrai quil est particu. à l'́́gard de cette édition et c'est à partir du travail du savant anglais 40 que le texte doit être étudié. A. G. Drachmann [13] (p. 11) en 1963 et B. Gille [200] (pp. 146-147) en 1980. Hui consacrent une petite notice. Autrement nous ne disposons guère (outre E. W. Marsden) que de l'article d'A. G. Drachmann [44] en 1977.

38 Pol. 22, 24-25.
39 Cf. Aristote, Ouestions de mécanique, éd. O. AprLT, Leipzig, 1888.
40 [14], pp. 66-103: texte grec, apparat critique, traduction anglaise et commentaire 
PHILON DE BYZANCE (III ${ }^{\circ}$ s. a.C.). L'oeuvre de Philon est abondante et variée. Il nous en est resté quatre traités techniques. Les Belopoiica (= Livre IV de la Syntaxe Mecanique) ont ete edités par Kochly et Rustow [10] en 1853 avec utile), . Diels et $\mathrm{E}$. Schrremm [46] en 1918. L'édition de référence est moujours celle d'E. W. Marsden [14] en 1971. Il existe également une traduction partielle en français par V. Prou qui accompagne son édition de la Chirobaliste d'Héron [47]. Les Poliorcetica (= Livre V de la Syntaxe Mécanique) ont été édités par R. Schönc [45] avec les Belopoiica, par H. Diels et E. Schramm en 1919 [48]. L'édition de référence est maintenant celle d Y. Garlan, à la suite de ses Recherches de polior cétique grecque [49] en 1974. Une traduction française partielle a été réalisée par A. de Rochas d'Aiglun en 1872 [50] 41, puis reprise par A. de Rochas d'Aiglun et préliminaire sur les différentes éditions des mécaniciens anciens et leurs notice Les Pneumatica ne sous sont parvenus qu'en arabe. Nous disposons des traductions de Carra de Vaux [52] et d'A. de Rochas d'Aiglun dans son livre sur la science des philosophes et l'art des thaumaturges dans l'Antiquité [53]. Des extraits ont égale ment été publiés par V. Rose en 1870 [54] et par W. Schmidt en 1899 à la fin du tome 1 des oeuvres d'Héron [64] ${ }^{42}$. Le traité des Clepsydres a également été transmis dans une version arabe, nous disposons d'une analyse de ce texte par Carra de Vaux en 181 [5]. Un index pour les Belopoilica et les Poliorcetica a été réalisé Byzance sont peu no nous venons de citer contiennent l'essentiel pour la partie militaire. On peut y ajouter le chapitre consacré à l'oeuvre de Philon par B. Gille dans ses Mécaniciens grecs 43, l'introduction de F. D. Prager [54 bis] et les articles d'Y. Garlan en 1973 [57] et de J. M. Spieser en 1986 [58].

ATHENEE LE MECANICIEN (I ${ }^{\mathrm{r} r}$ s. a.C.) n'a fait l'objet d'aucune édition ou étude récente à notre connaissance 44 . Nous citerons donc pour mémoire les éditions d'A. de Rochas d'Aiglun en 1884 [59], les études de F. Krohn [60], C. Cichorius [61] les passages qui lui sont consacrés dans l'étude de F. Lammert [62] et surtout dans celle de W. Sackur [63]; la question qui revient toujours dans ces travaux est celle de la relation entre les ch. XIII à XV du livre $\mathrm{X}$ de Vitruve et les p. 9 a 26 (Wescher) du Traite des Machines d Athénee puisque la correspondance entre ces deux passages est totale, sur le fond et sur la forme. Nous avons tenté une . de Vitruve ${ }^{45}$.

41 Pour reprendre les termes d'Y. GARLAN ([49], p. 287), cette édition: «vaut surtout
l'originalité et l'ingéniosité des vues que [Dr RocHAs] put avancer, en tant qu'ingénieur 12 Une belle édition des Preumatica [54 bis] a en outre été publié par F. D. PRAGER

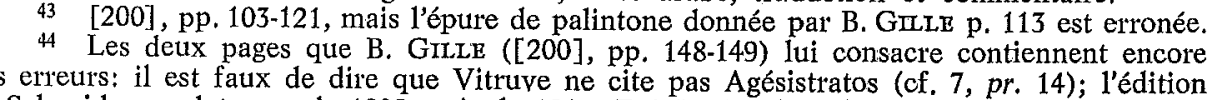

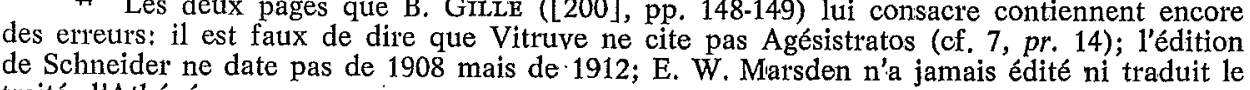

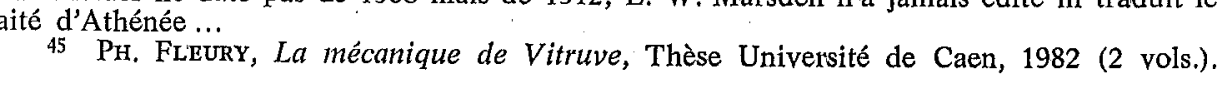

HERON D'ALEXANDRIE (Fin du Ier siècle p.C. ?) est le mécanicien grec le

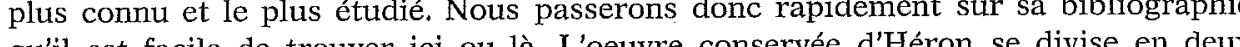
quill est les techniues civiles et les travaux scientifiques (Pneumatiques, Auto mates, Mécaniques Catoptrique, Métriques, Dioptre, Définitions, Géométrie, Stéréo. tomie, Mesures) d'une part, la mécanique militaire (Belopoiica, Chirobaliste) d'autre part. L'édition complète des traités de la première branche dans la collection Teubner [64] de 1899 à $1914^{46}$ permet de se passer des éditions partielles anté rieures. On pourra y ajouter l'édition des Metriques par E. M. Bruijns en 1964 [65] les extraits des Mécaniques édités dans la collection Loeb parmi les Greek Mathematics [66], les extraits de la Dioptre et des Mecaniques tradults par $A$. $G$. Drachmann [13]. Pour la partì mís cil suffit d'utiliser maintenant l'édition $d^{\prime} E$. W. Marsden [14] et la traduction allemande de la Chirobaliste, proposée par N. Gude et D. Baatz à la fin de leur article de 1974 sur les fragments de baliste trouvés à Gornea et Orsova [67. Toute étude sur Héron a interet a partir de louvrage de Th. Henri-Martin ${ }^{47}$. Il est vieilli mais reste malgré tout une base solide pour les recherches non seulement sur Herron dAlexandrie, mais sur tout le corpus mécanique grec. Parmi les études récentes, citons les articles de M. Federspie chapitre qui lui est consacré par B. Gille [200] pp. 122-145.

APOLLODORE DE DAMAS (déb. du I $\amalg^{\circ} s . p . C$ ) comme Athénée le Mécanicien a suscité peu de vocations chez les éditeurs et les commentateurs. Pour les éditions de sa Poliorcétique, nous devrons donc nous contenter des recueils de Wescher [11] et de Schneider [12], et de la traduction donnee par E. Lacoste en 1890 [71] à partir du texte de Wescher. Pour les études il faut utiliser celles B. Gille lui consacre quelques lignes: [200] pp. 161-162.

POLYEN (II s. p.C.) est l'auteur d'un traité de Stratagèmes dans la tradition qui est représentée à Rome par Frontin (cf. infra). Il s'agit en fait d'une compien huit livres dédiés aux emperenr guerre contre les Parthes $(162 p . C$.
en 1887 [72] et réimprimé en 1970 .

PAPPOS (III s. p.C.) est un auteur scientifique, mais le livre VIII de sa collection mathematique contient des éléments de mécanique, notamment des

46 Tome 1: Pneumatica et automata (grec-allem.) éd. W. SCHмтDT, 1899. Tome 2:

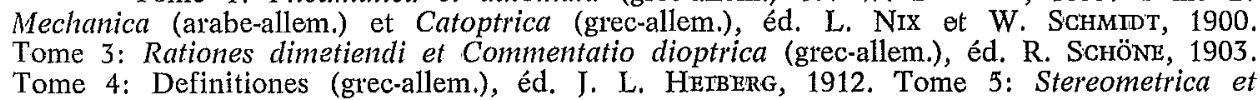

De mensurivis (grec-allem.), éd. . L. LertBeRG, 1914.

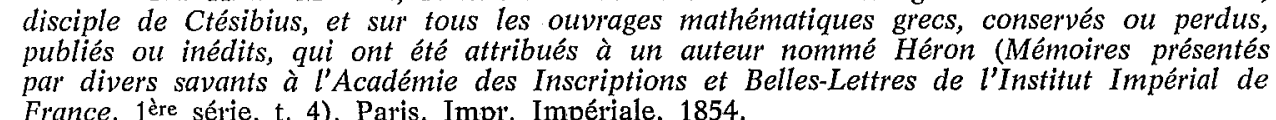


commentaires sur les engrenages et les démultiplications. Il a été édité et traduit en latin par F. Hultsch [73], traduit en français par P. Ver Eecke [74]. Quelques

\section{LITTÉRATURE LATINE 48}

CATON (II s. a.C.). Une concordance du De agricultura a été réalisée en 1983 par W. W. Briggs [75], un index en 1988 par G. Purnelle [75 bis]. Des éditions se trouvent dans les grandes collections: celle de W. D. Hooper et H. B. Ash pour Loeb [76], celle de A. Mazzarino pour Teubner [77], celle de R. Goujard pour la C.U.F. [78]. O. Schönberger a realise pour la collection Tusculum-Bücherei une édition complete en 1980 [7] avec, en plus du De agriculura, le texte et la traduction de tous les fragments connus jusquitc. Cetle cdition est accompagné P. Thielscher de 1963 [80]. Pour l'étude de la langue, nous disposons depuis 1970 du travail de S. Boscherini [81]. Signalons enfin deux articles récents: celui de L. Calboli Montefusco en 1980 [82] sur les ingrédients des recettes de patisserie et celui de E. Simovičova en 1983-1984 [83] sur les termes relatifs au travail agricole 49 .

CESAR (Irer s. a.C.). Il n'y a pas lieu ici de présenter la bibliographie césarienne. Nous signalons seulement l'index complet du corpus césarien de B. F. Schemann [84] paru en 1976, la concordine ét de W. Wimmel en 1973 sur les côtés techniques du siège d'Avaricum ${ }^{50}$ [86] et notre article en 1981 [211] sur la nomenclature des machines de jet romaines dans lequel nous essayons d'expliquer à propos des sièges de Marseille ${ }^{51}$ et d'Avaricum les emplois des mots balista, catapulta et scorpio.

VARRON (Ir s. a.C.). On trouvera la bibliographie antérieure à 1980 dans le Bericht de B. Cardauns [87]. Une concordance du De re rustica a été publiée en 1983 par W. W. Briggs [88]. Dans la Collection des Universités de France J. Heurgon L'étude de J. Kolendo en 1979 [91] porte sur les techniques permettant d'éconpmiser le temps de travail, celle de J. Collart, la même année [92], sur une comparaison entre le vocabulaire de Varron et celui de Pline.

VITRUVE ( Irr $^{\text {s. }}$ a. C.) est l'auteur technique sur lequel s'exerce actuellement la plus grande activité scientifique: depuis quelques années les colloques se succè-

48 Pour rétude de la tradition manuscrite d'une bonne partie des auteurs que nous
dén

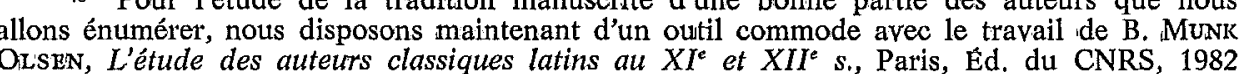
= catalogue des manuscrits classiques latins copies du $\mathrm{XI}^{\circ}$ au $\mathrm{XII}^{\circ} \mathrm{s}$.). instrumentum, agricola, colonus, pater familias, dominus, familia, factor, leguli,

strictores, capulatores.'
50 Gall. 7,13 sqq.
51 Ciu. 2 texte de Fensterbusch par l'équipe du Centre d'Etudes et de Recherches en Langue Anciennes de lUniversité de Caen [93] remplace l'index de Nohl fondé sur l'éditio des référest en árie accos 1983. La meilleure édition complète est la plupart celle de C. Fensterbusch 52 [94]. Dans la Collection des Universités de France, sont déjà édités: le livre I en 1990 par Ph. Fleury [95] avec une Introduction générale au De architectura, le livre VIII en 1983 par L. Callebat [96], le livre IX en 1969 par J, Soubiran [97], le livre X en 1986 par L. Callebat et Ph. Fleury [98]. Le livre III par P. Gros est sur le point de paraitre [99]. Toutes ces édition de la C.U.F. sont accompagnées d'un important commentaire indispensable pour comprendre un texte souvent difficile. Dans notre Introduction au Livre I, nous

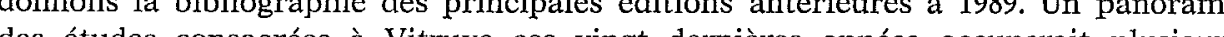
dizaines de panes, nous nous limiterons decc aux références postérieures à 1983, en commencant par les actes des Colloques consacrés directement ou indirectement au De architectura publiés depuis cette date: Colloque de Tours sur «Les traités architecture de la Renaissance» en 1981 [100], Colloque de Darmstadt consacié á Vitruve en 1982 [101], Colloque de Berlin sur le plan en 1983 [102], Colloque de Strasbourg sur "Le dessin d'architecture dans les sociétés antiques» en 1984 [103], Colloque de Londres en 1986 sur l'influence de Vitruve [104], Colloque de beyde enfin sur le De architectura et larchitecture hellénistique et répucrées à Vitruve: celle d'H. Knell en 1985 [106] et celle d'E. Romano en 1987 [107] trois autres laissent une large place au De architectura: celle de J von Freeden en 1983 [108] sur la «Tour des vents» d'Athènes ${ }^{53}$, celle de B. Wesenberg la même année [109] sur les sources litteraires concernant l'architecture grecque et celle d'E. Rawson en 1985 sur la vie intellectuelle à l'époque tardo-républicaine [110] dans laquelle elle inclut Vitruve. Meme en se limitant a la date de 1983 et en ne reprenant pas les references qui se trouvent dans les actes des colloques que nous de recher J. Adam sur l'utilisation de Vitruve et des gromatici notamment [111], E. Gabba sur la fondation d'une nouvelle Salapia mentionnée par Vitruve en I, 4,12 [112], C. Tosi sur l'implantation des temples dans le contexte urbain [113], W. Weber sur l'horloge à eau de 8,8 [114] J. I. Porter sur la scaenographia [115], M. Vickers sur les caryatides [116]; à propos de la posterité de Vitruve: G. Barbe Coquelin de Lisle sur l'Espagne de la Renaissance et Vitruve [117], P. Mylonas sur la fortune de Vitruve au Moyen Âge [118], des raisons d'écrire de Vitru de V. Novara [120]; sur les préfeces di ; a propos

52 Bien que l'apparat critique soit insuffisant et rédigé de façon très incohérente, l'edition du texte repose sur une collation complète et sérieuse des principaux manuscrits: 54 Cat article est en fait une reprise de J. ANDRÉ, «Le prologue scientifique et la
hétorique: les préfaces de Vitruve», $B A G B$, 1985, pp. 375-384. 
VIRGILE (Irer s. a. C.). Les Géorgiques ont profité, comme les autres oeuvres virgiliennes, d'un regain de faveur à l'occasion du deuxième millénaire de la mor du poète en 1981. W. Suerbaum [122] leur a consacré une bibliographie spécial en 198, on sé en 1956 une édition pour la C.V.F. [123]. Deux éditions particulières celle d'A. Barghesi en 1980 [124] et celle de M. von Erren en 1985 [125]. Une mońo graphie a été consacrée aux Géorgiques par G. B. Milles en 1980 [126]. Parmi les articles récents nous n'avons retenu que ceux portant sur la nature du traite (est-ce un traite technique ou une oeuvre poetique avant tout? - ses rapports avec la poésie diclactique: Hesiode, Lucrèce-ses rapports avec la litterature agrono mique: Caton, Varron-lidéologie), ceux portant sur un point technique et enfin les étules de Vocalor A. Cossarini compare Caton, Varron, Virgile et Columelle sur l'idéologie de la propriété [128], R. Martin s'interroge sur le sens du traité et considère que seuls les livres I et II méritent le titre de Géorgiques et correspondent au poème d'Hésiode [129], G. Kromer note l'influence d'Hésiode, Lucrèce et Aratus sur Virgile [130], J. A. Fernandez Delgado montre en sens inverse le profit qui peut être tiré de Virgile pour interpréter Hésiode [131], F. Muecke [132], A. Grilli [133], A. Marastoni [134] et M. S. Spurr [135] s'interrogent sur le sens du traité avec awons relevé l'article de G. Forni à propos de l'araire [136] et sur le vocabunise celui d'O. Pasqualetti à propos du mot ingenium [137].

COLUMELLE ( ${ }^{\text {er }}$ s. $p . C$.). Dans un volume d'Aufstieg und Niedergang de römischen Welt paru en 1985, R. Martin a fait le point bibliographique [138] En 1971 G. G. Betts et W. D. Ashworth ont produit un index [139] fondé sur l'édition d'Uppsala ss. L'edition complète la plus recente est, à notre connaissance, celle de W. Richter [40] en 1981-82. Dans la Collection des Universités de France De arboribus en 1986 [142]. Parmi los Ćtudes récentes: G. Maguilli en 1980 ser le vocabulaire non virgilien du livre X [143], P. Sáez Fernández en 1983 sur un système d'extraction de l'huile [144], P. P. Corsetti en 1986 sur la tradition manuscrite [145].

APICIUS ( (re $^{\text {er }}$ p. C.). Le traité sur l'art culinaire d'Apicius est un peu à part cans la litterature technique car pour le reste les techniques de transformation de la nourriture nous ont ete transmises par les traites des agronomes, mais nous savons quill existait une littérature cullnare grecque et romaine ${ }^{36}$. Le De coquinaria a été édité chez Teubner en 1969 par $M$. E. Milham [146], et da

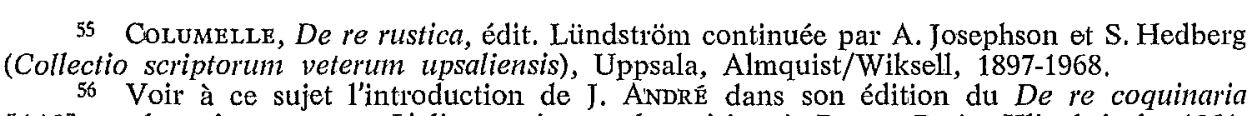

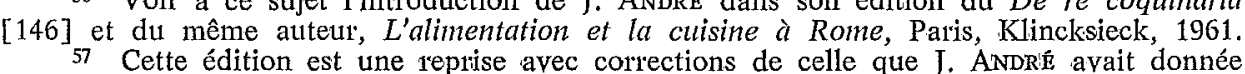
chez Klincksieck en 1965. des noms d'instruments de cuisine rencontrés chez Apicius et chez Pétrone [148] et $\mathrm{F}$. Marchese a étudjé la langue des recettes [149].

FRONTIN ( ${ }^{\text {or }}$ s. p.C.) a écrit dans trois domaines techniques différents: l'adduction d'eau, les stratagèmes et l'arpentage. L'index de J. Costas Rodriguez en 1985 [150] vient prendre la suite de celui de G. Bendz en 1939 pour les Strategemata. Pour le De aquis on pourra utiliser l'edition de P. Grimal dans la C.U.F [151] en 1961, ou celle de C. Kunderewicz chez Teubner [152] en 1973 58, pour les Strategemata la seconde édition de G. Bendz en 1978 [154], et pour le 1984 [155]. Dans la Leb Classical Library les Strateremato et le De aquis sont édités ensemble par C. E. Benett [156]. En 1983 la FRONTINVS-Gesellschaft a consacré un beau livre à l'alimentation en eau dans la Rome antique [278], on y trouve une traduction nouvelle du De aquis par G. Kühne à partir du texte de C. Kunderewicz. En 1983 F. Hernández-González a étudiê le vocabulaire relatif à l'eau dans l'oeuvre de Frontin [157].

PLINE L'ANCIEN (Ir s. p. C.) n'est pas un auteur technique à proprement parler mais son encyclopédie est parsemée d'indications sur les techniques sur l'agriculture). Comme pour César, il n'y a pas lieu de dresser ici la biblio graphie plinienne; cela a du reste été fait par G. Serbat dans un volume d'ANRW publié en 1986 [158]. Une concordance de la Naturalis Historia par P. Rasumek et $D$. Najock est en principe sous presse chez Olms. Parmi les etudes récentes sur des points techniques, voir G. Forni [159] et J. Kolendo [160] sur l'araire, H. Loeber [161] sur la tabrication du verre. Rappelons aussi la comparaison nous avons citée à propos de ce dernier.

HYGIN (II'-III ${ }^{\circ}$ s. p. . C. ?) 59 . Nous ne savons rien de l'auteur du De munitionibus castrorum. Il ne doit pas être confundu avec l'Hygin du I I ${ }^{\text {er }}$ s. a.C. bibliothécaire d'Auguste, auteur d'un traité sur l'astronomie, mais il ne s'agit sûrement pas non plus de lauteur du De limitibus et du De condicionibus agrorum celles d'A. Grillone chez Teubner [162] et de M. Lenoir dans la C. U. F. [163], Les règles de la collection Teubner ne permettant pas de commentaire. publié ses réflexions sur l'ouvrage dans plusieurs articles: [164] [165] [166]. E. Birley s'est également penché sur le difficile problème de la datation de l'ouvrage [167] (peut-être vers 160-170 p.C. selon lui).

CETIUS FAVENTINUS (III s. p.C.) résume le De architectura de Vitruve mais utilise aussi d'aur

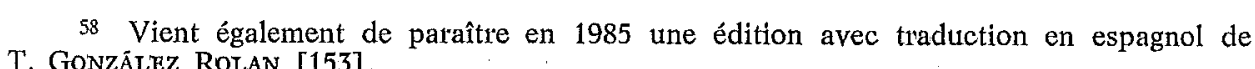
59
59
Début II I'

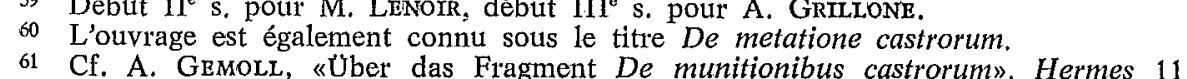
1877, pp. $164-178$. 
sur Cetius Faventinus, Palladius et la science édilitaire romaine [168]. Voir aussi solaires décrits par Faventinus [169].

AMMIEN MARCELLIN (IVes. p.C.). Du point de vue de la mécanique milniveau que César. Ce nest pas un technicien mais ses notices sont bien informées: au livre XXIII le chapitre IV est entièrement consacré à la description des machines de siege: baliste, scorpion ou onagre, bélier, hélépole et massettes. Le livre XXIV par alleurs contient plusieurs mention précises de l'emploi des machines de guerre 2 ; une bibliographe dhe an travo d'indexation sont parus à deux ans dintervalle ...: celui de $M$. Chiabo en 1983 [171] et celui de L. Viansino en 1985 [172]. Une concordance sur microfiches a par ailleurs été établie en 1980 par G.J.D. E. Archbold [173]. Pour not point de vue l'édition du livre XXIV avec commentaire par J. Fontaine dans C.U.F. est la plus utile [174]. Voir aussi le livre de N.J.E. Austin sur les connais sances militaires d'Ammien [175], l'édition commentée d'E. W. Marsden que nous avons déjà citee [14] et, contre le travail d E. W. Marsden, Yartele de M. F. A. Brok en 1977 [176] qui traduit et commente la descique complete mais a voulu conclure sionner son lecteur et a choisi les détails les plus propres à cela. Plusieurs des conclusions de M. F. A. Brok sont contradictoires avec celles d'E. W. Marsden.

DE REBVS BELLICIS ( $\mathrm{IV}^{\circ}$ s. p.C.). La date de ce traité est controversée mais si l'on met à part les hypothèses extrêmes de R. Schneider ${ }^{\circ 3}$ (XIV s. p.C.) et de R. Neher (regne de Justhien: 527.565), toutes les datations proposés vont lu règne de Cour de l'édition avance une hypothèse précise: le traité aurait été dédié à Valens à la fin de 368 ou au début de 369. L'édition de R. I. Ireland est suivie d'un index uerborum malheureusement entaché d'erreurs et d'oublis que nous avons corrigés dans notre compte-rendu pour la Revue de Philologie ${ }^{6}$. Le De rebus bellicis connaît un regain d'intérêt et les études qui lui ont été consacrées ces vingt dernières années sont relativement nombreuses. Deux editions commentees ont vu le jour: celle de S. Condorelli en 171 [178] et celle que $M$. W. C. Hassall et $\mathrm{R}$. I. Ireland avaient présentée ensente dition ouvrage sur la littérature de re militari [212] (p. 144 sqq.). H. Brandt a écrit une monographie [180]. La plupart des articles ont abordé l'inévitable question de la
datation: A. Cerati [181], S. Mazzarino [182], B. Baldwin [183], F. Kolb [184] [185],

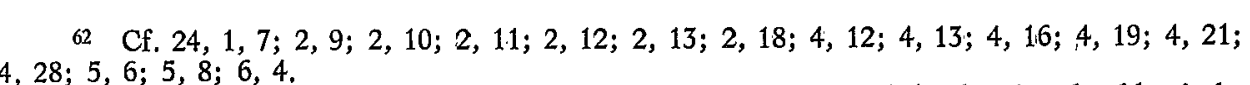
, 28,

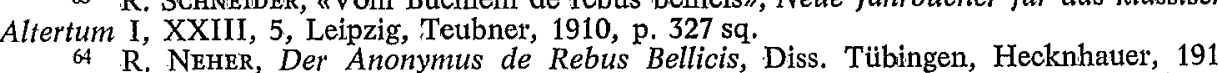
6 . R. NEHRR, Der Anonymus de Rebus Bellicis, Diss. Tübingen, Hecknhauer, 1911. au
Inventor Oxford, Clarendon Press, 1952 . On se méfiera donc des risques de confusion dans les citations que cette innovation va nécessairement entraîner. G. Bonamente [186], A. E. Astin [187], D. Foraboschi [188]; I. G. Maier [189] et [190] nombre de termes ${ }^{67}[191]$.

VEGECE (fin $\mathrm{IV}^{\text {e }}$ s. p.C. ou début du $\mathrm{V}^{*}$ ?). R. Sablayrolles [192] vient de faire une excellente bibliographie de Végèce. Nous ne pouvons que renvoyer à son travail bien présenté en trois parties: I-Éditions. II - Traductions. III - Commentaires. Dans ce troisième volet R. Sablayrolles passe d'abord en revue les articles et les ouvrages qui traitent de la date de composition (fourchette comprise entre 383 et 410 p. C.), puis ceux qui traitent de la nature et de lintluce de l'oeuvre (est-ce un (Can techniques de l'oeuvre. L'édition complète de référence de l'Epitoma rei militaris reste la deuxième édition de C. Lang chez Teubner en 1885 [193].

MODESTUS (III s. p.C. ou postérieur à Végèce?). Nous plaçons à côté de Végèce le Libellus de nocabulis rei militaris de Modestus car il est très proche du livre II de l'Epitoma rei militaris. Si l'on en croit la dédicace, il est adressé à son livre II mais comme Modestus n'est pas cité une seule fois dans l'Epitome alors que pour le reste Végèce donne volontiers ses sources, il est possible aussi que la dédicace soit apocryphe et que l'opuscule ne soit qu'une copie tardive du Livre II de l'Epitoma rei militaris.

PALLADIVS (début $\mathrm{V}^{\text {e }}$ s. p.C.). L'édition de référence pour l'oeuvre complète de Palladius est maintenant celle de R. H. Rodgers, parue en 1975 chez Teubner [194]. Elle est suivie d'un index nominum, rerum et uerborum et contient une bibliographie (pp. XXIII-XXVII). Pour les livres I et II l'édition de R. Martin dans arécédée en offre l'avantage d'être accompagnée d'un commentaire [195]. Elle est precédée en outre d'une importante introduction sur Palladius et son oeuvre 68. quente sur la tradition manuscrite de Palladius [196]. A pub près en même temps
quate que ces deux éditions est parru un article de Ch. Josserand à propos du passage sur la moissonneuse gauloise [197]. Depuis, peu de choses à notre connaissance sur renseignements qui peuvent être tirés de l'Opus agriculturae pour l'étude de la vie rurale au Bas-Empire [198].

III. ÉTUDES SUR LES TEXTES TECHNIQUES

Selon les mêmes critères que ceux définis en introduction, nous voudrions un ensemble d'auteurs techniques, sur la langue ou le style des textes techniques ${ }^{\circ}$.

67 ballista quadrirotis, tichodiphrus, clipeocentrus, plumbata tribulata, plumbata mamil-

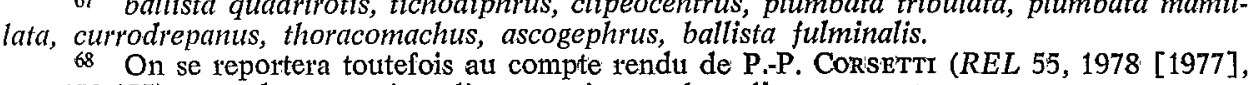
p. 452-455) pour la correction d'un certain nombre d'erreurs. 69 Pour la période qui va des origines de Rome à la fin de la République, F. HINard
s'est livré à un recensement des ouvrages partus entre 1983 et 1987 (les articles sont exclus, 
Etudes générales.

Rappelons d'abord que le vieil ouvrage d'H. Blümner sur la technologie et la terminologie des arts et métiers dans les textes grecs et romains 70 a été réim-
primé à New-York en 1979 chez Arno Press. Il est toujours utile pour bien des questions de terminologie et nous pourrions aussi le citer dans le chapitre suivant, sur les études de contenu. Dans une thèse de 1975 sur la littérature romaine spécialisée, B. Deinlein [199] étudie successivement les formes de la littérature didactique dans lAntiquité (ouvrage d'initiation, traité systématique, lettre, dialogue, poème ...), la forme de l'ouvrage spécialisé romain et la pratique des exordes.

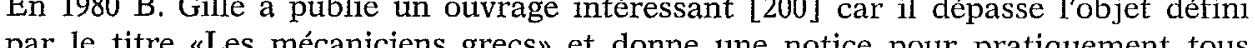
les mécres deux derniers chapitres sur «le blocage» et «la formation de la connaissance technique» présentent des hypothèses nouvelles sur les problèmes de la stagnation technique dans l'Antiquité avec une réfutation de la plupart des anciennes théories. Malheureusement ce travail est entaché d'un grand nombre d'erreurs: contradictions de dates, erreurs de conversions de mesures, conception tout à fait erronée des machines de jet, affirmations contestables sur l'oeuvre de Vitruve ... Dans son livens sur les alingue techiche dol lathon, C. de Meo [201] prend "technique» au culture, la langue juridique, la langue sacrale et la langue militaire puis rassemble dans un même chapitre le langage politique, la langue de la médecine, celle de l'astronomie et de l'astrologie, celle enfin de la mer- et de la navigation. Chaque chapitre est suivi d'une bibliographie assez fournie, mais l'ensemble reste de portée générale.

Le chapitre consacré aux problèmes du vocabulaire technique par J. Dubois dans le recueil d'A. Rey sur la lexicologie [202] n'est pas spécialisé pour les langues anciennes mais il contient des remarques intéressantes sur la genèse d'un nomisation. J. André, lui, s'est penché précisément sur la constitution des langues techniques en latin $[2]$

b. Etudes spécialisées dans un domaine.

ARCHITECTURE ET CONSTRUCTION. Le Dictionnaire méthodique de l'arch tecture grecque et romaine de R. Ginouvès et R. Martin [203] dont le premier

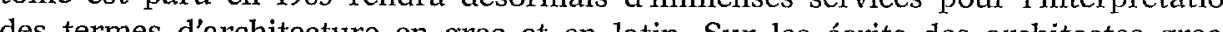
des termes d'architecture en grec et en latin. Sur mais quelques renvois sont faits occasionnellement). Dans la première partie de son travail,
deux rubriques intéressent notre sujet: celle consacrée aux sourtese et celle consacrée aux
sciences et techniques: F. HENARD, «Rome des origines à la fin de la République», $R H$ ant, sciences et techniques: F. HENARD, «Rome des origines à la fin de la République», RH 561 ,
janv.-mars 1987, pp. 121-165. 70 H. BLÜMNNR, Technologie und Terminologie der Gewerbe und Künste bei Griechen
und Römern, Leipzig, Teubner, vol. I: 1875, vol. HI: 1879, vol. III: 1884, vol. IV: 1887 .
AGRICULTURE. L'ouvrage de M. G. Bruno, Il lessico agricolo latino, paru en 196971 [206] n'apporte rien de plus que le Thesaurus Lingulae Latinae ou le Dicclurs de traductiog et les classements sont souvent aberrants. Quant à l'ouvrage de S. Andrei [207], il est trop imprécis et manque de références pour être un véritable outil scientifique.

Pour l'étude des auteurs agronomes, K. D. Withe a fait un excellent point de la question dans Aufstieg und Niedergang der Romischen Welt [208] tout au moins 2009] bien que celui-ci n'envisage pas l'aspect technique de la question.

GUERRE. Sur le vocabulaire des stratagèmes en latin et en grec, voir E. L. Wheeler [210]. Sur la nomenclature des machines de jet romaines, voir Ph. Fleury [211]. L'ouvrage de V. Giu
surtout sous ses aspects juridiques.

\section{IV. ÉTUDES SUR LE CONTENU TECHNIOUE}

Nous terminerons ce panorama des études sur la littérature technique ces vingt dernières annees en citant, de façon nécessairement rapice, les ouvrages sur les données littéraires, mais aussi sur les données archéologiques, iconographiques, épigraphiques... Ces travaux sont un complément indispensable pour une parfaite compréhension des textes. Il existe plusieurs instruments bibliographiques auxquels on se reportera pour les études anciennes; voir par exemple
R. J. Forbes [213], F. Russo [214], E. S. Ferguson [215], J. P. Oleson [216].

a. Etudes générales sur les techniques de l'Antiquité.

Les «Histoires des Techniques» sont nombreuses. L'Histoire Générale des Techniques sous la direction de M. Daumas [3] est désormais un classique (comme son logy sous la direction de C Singer, E J. Holmyard, A. R. Hall et T. I. Williams [217] a été réimprimée une nouvelle fois en 1972. En 1978 est parue dans l'Encyclopédie de la Pléiade l'Histoire des Techniques sous la direction de B. Gille [218], en 1984 Thistory of Engineering de D. Hill [219].

Parmi les ouvrages que nous citons sous les références [220] à [227] se dégage nettement la monographie récente d'un spécialiste des techniques anciennes: K. D. White, Greek and Roman Technology [226] qui toutefois ne traite pas la technologie militaire, si ce n'est dans l'appendice sur le développement de la cataR. Harot [229]. Sur la question des rapports entre technique et société que nous

1 Il sagit en fait de la reprise de deux articles parus antérieurement: M. G. BruNo,

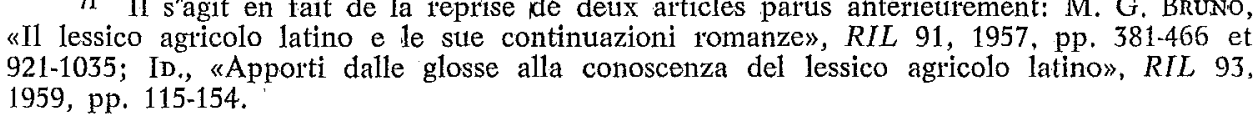


n'abordons pas directement ici, voir par exemple le livre de D. Furia et P. Serre ${ }^{72}$ et l'article de V. de Magalhães-Vilhena ${ }^{73}$.

b. Etudes spécialisées sur le contenu technique.

Nous avons rangé les références [229 bis] à [285] sous quatre rubriques: architecture ${ }^{74}$ et construction agriculture ${ }^{3}$-guerre - adduction d'eau et machines pour extraire l'eau; les références de cette derniere rubrique auraient pu être réparties entre construction et agriculture, mais nous avons préféré les traiter à part car années. A l'intérieur de chaque rubrique nous avons cité dans l'ondre chnieres gique, d'bord les ouvrages puis les articles.

$$
* *
$$

Il apparaît donc, au terme de cette revue, qu'une intense activité scientifique s'est exercée ces dernières annés dans le domaine de la littérature technique grecque et romaine; mais il est évident aussi que les efforts n'ont pas été également répartis. Certains auteurs connaissent plusieurs éditions successives alors que d'autres sont delaissés depuis un siècle. Vitruve par exemple est un auteur "surétudiés et c'est une bonne chose car son influence a été immense sur toute l'Europe a l'epoque de la Renaissance. Des auteurs comme Enée le Tacticien, parler des également intéressé les chercheurs. Par contre le pseudo-Aristote, Biton ...) on le Mécanicien, Apollodore de Damas n'ont pratiquement pas été étudiés. Végèce dont l'influence dans le domaine militaire au Moyen Âge et à la Renaissance a été comparable à celle de Vitruve dans le domaine architectural n'a été l'objet que d'une activité moyenne, comme en temoigne la bibliographie de R. Sablayrolles [192] et surtout il na pas connu dedition scientifique complète depuis la fin du XIX siècle. Une des priorités de la recherche ces prochaines années devrait donc être

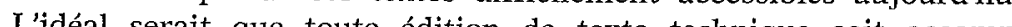

Lideal serait que toute édition de texte technique soit accompagnée d'un qui permettaient au lecteur antique de comprendre et d'apprécier rérérences était dit. L'usage de certaines collections, comme Teubner, ne le permet pas et il est dommage que la Collection des Universités de France, qui se distinguait jusqu'alors par un riche commentaire pour les textes techniques, soriente vers la limitation de celui-ci $\%$. La publication dans un ouvrage séparé ou dans des
articles de revue du travail d'«explication», que dans tous les cas les éditeurs

72 D. FURIA et P. SRRRE, Techniques et sociétés, Paris, Colin, 1970, t. 1. a. DE MAGALHÃRS-VirHENA, «Essor scientifique et technique et obstacles sociaux

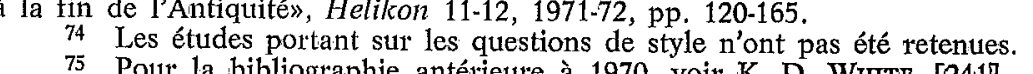

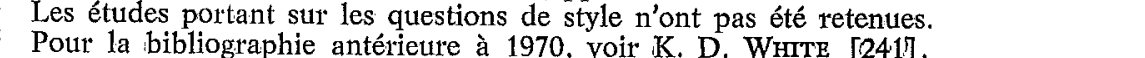

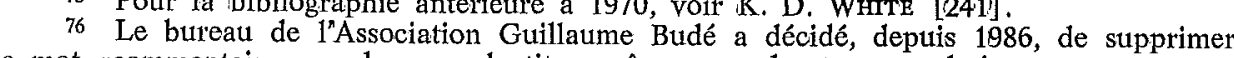

de ce genre d'oeuvre doivent faire pour pouvoir proposer une traduction conve Lun

ente dans les études. Alors qu'il y a pléthore d'études générales sur les techniques de l'Antiquité, sur l'histoire des techniques 77, nous vocabulaire, sur les machines ou les instruments qu'ils décrivent. Ces travaux font appel à l'association de compétences diverses (philologiques, techniques, paléographiques, archéologiques ...) et là encore il ne peut y avoir de cloisonnement entre disciplines.

Notre étude pourrait enfin être complétée par un recensement des fragments techniques et des travaux qui leur sont consacrés ainsi que par un inventaire rait la perspective au-delà des oeuvres conservées sur une certaine étendue et pergmettrait de mieux cerner ce qu'était la véritable langue technique, celle employée par les techniciens et les hommes de métier.

\section{LISTE DES REFGRENCES}

0. Géneralités ET RECUEILS

[ 1 ] J. Beaujeu, "La littérature technique des Grecs et des Latins», Actes du Congrès de Grenoble de l'Association G. Bude (1948), Paris, Belles-Lettres, 1949, $21-79$.

[ 2 ] J. ANDRÉ, "Sur la constitution des langues techniques en latin", Etudes de Lettres. Revue de la Faculté des Lettres de Lausanne, Janvier-Mars 1986 ("Sciences et techniques à Rome»), 5-18.

[ 3 ] Historre Générale des Techniques, publiée sous la direction de M. Daumas, tome I: Les origines de la civilisation technique, Paris, PUF, 1962.

[ 4 ] J. J. HaLL, «Was Rapid Scientific and Technical Progress Possible in Antiquity?», Apeiron 17, 1983, 1-13.

[ 5 ] J. Galliard et R. Martin , Les genres littéraires à Rome, Paris, Scodel, 1981.

[ 6 ] A. Daıs, "Les stratégistes byzantins» (texte mis au net et complété par J. A, De Foucaurr), Travaux et Mémoires du Centre de Recherches de Civi-

[ 7 ] Scriptores rei rusticae neteres latini, ed. J. M. GEsNER, Leipzig, 1735

[ 8 ] Scriptores rei rusticae ueteres latini, ed. I. G. SchNEIDER, Leipzig, 1794-1797.

[ 9 ] Veterum mathematicorum, Athenaei, Bitonis, Apollodori, Heronis, Philonis et aliorum opera, graece et latine pleraque nunc primum edita ex manu-
scriptis codicis bibliothecae regiae, par M. THEvENor, Paris, Ex typographia
regia, 1693 . regia, 1693.

$\pi$ Il suffit pour s'en convaincre de constater le nombre d'ouvrages parus depuis la
dernière guerre en toutes langues et qui portent le titre «Histoire des techniques»... 
[10] H. Kochuy et W. Rusrow, Griechische Kriegsschriftsteller, Leipzig 1853. 11] C. Wescher, Poliorcétique des Grecs, Paris, Imprimerie Impériale, 1867.

[12] R. SCHNEDER, Griechische Poliorketiker (Abhandlungen der königlichen Gesellschaft der Wissenschaften zu Göttingen. Philologisch - Hist. Klasse, Neue
Folge, Bd. X, 1; XI, 1; XII, 5), Berlin, Weidmann, 1908-1912 (3 vols.).

[13] A. G. Drachmann, The Mechanical Technology of Greek and Roman Antiquits. A study of the literary sources (Acta hist. scient. nat. et 'med., 17), Copenhague, Munksgaard, 1963.

[14] E. W. Marsden, Greek and Roman Artillery. Technical Treatises, Oxford, Clarendon Press, 1971

[15] Les ALchimistes Grecs, tome I: Papyrus de Leyde, Papyrus de Stockholm, Fragments de Recettes, éd par R. Harrexu, (C. U.F.) Paris, Les Belles Lettres, 1981 .

[16] R. HALLEUX, «Techniques et croyances dans les recettes antiques et médié vales de sidérurgie», Caesarodunum 22, 1986, 114-128.

\section{LITTÉRATURE GRECQUE}

Hésiode

[17] M. HoFrnger et M. MUND-Dopchie, Lexicon Hesiodeum cum indice inuerso, Leiden, Brill, 4 fascicules à partir de 1975.

18] M. Hopinger et D. Pinte, Lexicon Hesiodeum cum indice inuerso. Supplementum, Leiden, Brill, 1985

[19] W. W. Mrnton, Concordance to the Hesiodic Corpus, Leiden, Brill, 1976

[ 20$]$ J. R. TEвBEN, Hesiod-Konkordanz, Hildesheim, Olms, 1977.

[21] Hésiode, Carmina, éd. A. Rzach, Stuttgart, Teubner, 3e éd. 1913 (réimp. 1958).

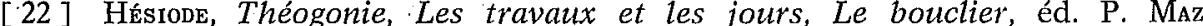
(C. U.F.), Paris, Les Belles Lettres, 1928 (réimpr. 1986)

[23. Hústoone, Theogonia, Opera et dies, Scutum, éd. F. Solmsen, (Scriptorum classicorum bibliotheca oxoniensis), Oxford, Clarendon Press, 1970.

[24] Héssoobs; Works and days, éd. avec introd. et comment. par M. L. WEst,

[25] J. MENDES DE CASTRo, «O filósofo-lavrador da Beócia, tradução e notas aos vv. 387-447 de Os trabalhos e os dias, de Hesiodon, Clássica 9, 1982, 15-25.

[26] G. P. Edwarns, The Language of Hesiod in its Traditional Context (Publi-

[ 27 ] M. ERREN, «Die Geschichte der Technik bei Hesiod», Festschrift für W. MARG zumn 70. Geburtstag, hrsg. von G. Kurz, D. MuelLer und W. NicolaI, Munich,
Beck, 1981, 155-166.
[28] G. Derenzins, «Una fonte per l'iconografia e la nomenclatura greca degli attrezzi agricoli. I codici esiodei), Studi e notizie IV dic. 1978 (Centro di

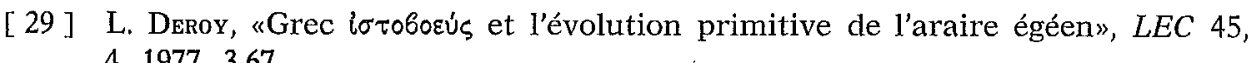
4, 1977, 3-67.

[ 30 ] J. Fuzier et P. Ghiron-Bistagne, «A propos d'un passage d'Hésiode. Technique de battage du blé et danses prédramatiques», CGITA 1, 1985, 19-22.

[ 31 ] F. BURANELLI, «Utensili per la lavorazione del legno in due tombe villanoviane da Veio», ArchClass 31, 1979 [1981], 1-17.

[32] J. J. Richardson and S. Piggorr, "Hesiod'wagon. Text and Technology», JHS $102,1982,225-229$

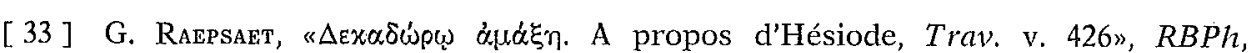
1987, 21-30.

[34] G. Ausac, «Le calendrier agricole dans les Travaux et les Jours d'Hésiode», Pallas 29, 1982, 3-15

\section{Énée le Tacticien}

[35] D. Barends, Lexicon Aineium, A lexicon and index to Aeneas Tacticus' military manual, On the defense of fortified positions (Diss. Utrecht), Assen, van cum, 1955

[36] Enée le Tacriclen, Poliorcétique, éd. par A. Dain, trad. par A. M. Bon, (C.U.F.), res, 1967

[37] M. BeтtaLL, «Enea Tattico e l'insegnamento dell'arte militare», AFLS 7, $1986,73-89$.

[ 38 ] R. Urban, «Zur inneren und äusseren Gefährdung griechischer Städte bei Aeneas Tacticus», Studien zur alten Geschichte. S. Lauffer zum 70. Geburt[39] Pн. Patrenden, «When did a guard duty end? The Regulation of the Night

\section{Pseudo-Aristote}

[40] Ps. Aristore, Mechanical Problems (Minor Works, pp.330-411), tr. by W. S. HETT (Loeb Classical Library), Londres/Cambridge (Mass.), Heinemann/ Harvard Univ. Press, 1936 (réimpr. en 1955)

[41] M. GaLitan, «Sur les problèmes mécaniques attribués à Aristote» (Congrès des Sciences, $5^{\circ}$ édit:: Histoire des Sciences, Paris, Colin, 1901, 101-111. [42] L. Sprague de Camp, The Ancient Engineers, Londres, 1960 (trad, allem. par
R. Rirscher, Die Ingenieure der Antike, Düsseldorf Econ., 1964). Biton [43] E. Schramm et A. Rehm, «Bitons Bau von Belagerungsmachinen und Ge
schüitzen», $A B A W$ NF II, 1929. 
[44] A. G. Drachmann, «Biton and the Development of the Catapult», Mélanges

\section{Philon de Byzance}

[45] PHILONIS mechantcae syntaxis libri quartus et quintus, éd. R. SchöNE, Berlin, 1893

[46] H. Diels et E. Schram, «Philons Belopoiica (viertes Buch der Mechanik)», Abhandlungen der Preussischen Akademie der Wissenschaften, PhilosophischHistorische Klasse, 1918, 16.

[47 ] V. Prou, «La chirobaliste d'Héron d'Alexandrie», Notices et extraits de la Bibliothèque nationale, 26, 2 (Paris, 1877), 1-319.

[ 48 ] H. Diels et E. Schramm, «Exzerpte aus Philons Mechanik, Buch. VII und VIII (vulgo fünftes Buch)", Abhandlungen der Preussischen Akademie der Wissenschaften, Philosophisch-Historische Klasse, 1919, 12

[49] Y. GaRLan, Recherches de poliorcétique grecque, (BEFAR 223), Paris, De Boccard, 1974.

[50] A. DE Rochas n'Arguun, Traité de fortification par Philon de Byzance, Paris, 1872

[51] A. de Rochas d'Atgiun et $C_{H}$, Graux, «Traité de fortification par Philon de Byzance», RPh 3, 1879, 91-151.

[52] Philon de Byzance, «Le livre des appareils pneumatiques et des machines hydrauliques de Philon de Byzance, édité d'après les versions arabes d'Oxford et de Constantinople et traduit en français par le Baron CARRA DE VAUX, membre du conseil de la Sociéte Asiatique de Paris (Notice, texte arabe, traduction)", Notices et extraits des manuscrits de la Bibliothèque

53 ] A. DE Rochas D'Arguun, La science des philosophes et l'art des thaumaturges d'ans liantiquité, Paris, Masson, 1882 (2 édit. Paris, Dorbon-Ainé, 1912; réimpr.

[54] Anecdota graeca et greco-latina, éd. V. Ross, Berlin, 1870, t. 2

[ 54 bis] Philon de Byzance, Pneumatica, éd. F. D. Prager, Wiesbaden, L. Reichert,

[55] Philon de Byzance, «Clepsydres. Analysé par Carra de Vaux d'après une tradition arabe», Journai asiatique, 1891 , t. 1, p. 295.

56] M. ARnrm, Index verborum a Philone Byzantio in mechanicae syntaxis libris quarto quintoque adhibitorum, Leipzig, Teubner, 1927 (réimpr. Hildesheim,

] Y. Gardan, «Cités, armées et stratégie à l'époque hellénistique d'après l'oeuvre

[ 58 ] J. M. SPresser, «Philon de Byzance et les fortifications paléochrétiennes», La fortification dans l'histoire du monde grec. Actes du Colloque interet sociale du monde et sociale du monde grec» (Valbonne, déc. 1982), éd. par P.
H. Treziny. Coll. int. du CNRS. Paris, Ed. du CNRS, 1986, 363-368.
Athénée le Mécanicie

[59] AThénée le Mécanicien, «Traité des machines. Trad, française par A. de Rochas D'Arglun,, Mélanges GRAUX, Paris, 1884, 781-801.

[60] F. Квонм, Quaestiones Vitruvianae, Particula II: De Vitruvio auctore commentarii qui inscribitur' 'A $\theta$ n'valion $\pi$ sph

[61] C. Crcновıus, «Das Werk des Athenaeus über Kriegsmaschinen», Römische Studien-Studia Historica, 80, $2^{\circ}$ édit., Berlin/Leipzig, 1922, $271-279$ (réimpr.

[62] F. Lammert, «Die antike Poliorketik und ihr Weiterwirken», Klio 31, 1938

[63] W. SAckuR, Vitruv, Technik und Literatur. Vitruv und die Poliorketiker. Vitruv und die Christlicheantike. Bautechnisches aus der Literatur des
Altertums, Berlin, W. Ernst, 1925.

\section{Héron d'Alexandrie}

[64 ] HERONIs Alexandrini opera quae supersunt omnia, Leipzig, Teubner, 1899-1914 $(5$ vols.)

[65] Héron d'Alexandrie, Metrica, éd. et trad, en anglais par E. M. Bruijns, Leyde, 1964.

[66] GREEK MATHEMATICS. Selections Illustrating the History of Greek Mathematics, ed. I. Thомаs, Lóndres/Cambridge (Mass.), W. Heinemann/Harvard

[67 ] N. Gudea et D. BAATZ, «Teile spätrömischer Ballisten aus Gornea und Orsova (Rumänien), mit einem Anhang: Herons Cheiroballistra (Ubersetzung)

] M. FedERSPIEL, «Sur un passage des Definitiones du Ps.Héron d'Alexandrie (éd. Heiberg, p. 156, 1.1-5)» RHS 32, 1979, 97-106.

[69] W. J. Verdenius, «Opening doors again», Mnemosyne 33, 1980, 175

[70] F. KRAFFT, «Heron von Alexandria», Exempla historica. Epochen der Welt geschichte in Biographien, t. 5: Forscher und Gelehrte, Frankfort, Fischer

Apollodore de Damas

[71] «Apolzodore de Damas. Poliorcétiques. Trad. par E. Lacoste», REG 3, 1890, 231-281.

Polyen

[72] Polyen, Strategematon libri VIII, éd. E. Wörffun et I. MeLber (suivi de Incerti scriptoris byzantini liber de re militari, éd. R. VARI), Stuttgart, 
Pappos d'Alexandrie

[73] PAPPI Alexandrini Collectionis quae supersunt, éd. F. Hulrsch, Berlin, Weidmann, 1875-1878 (3 vols).

[74] Pappos d'Alexandrie, La collection mathématique, trad. par P. Vвr Еecке, Paris/Bruges, Desclée de Brouwer, 1933 (2 vols.)

\section{LITTÉRATURE LATINE}

Caton

[75] W. W. Brigas, Concordantia in Catonis librum De agricultura, Hildesheim, Olms-Weidmann, 1983.

[ 75 bis ] G. Punnelus, Cato. De agricultura. Fragmenta omnia seruata. Index
uerborum. Liste de fréquence. Relevés grammaticaux, Liège, C. I.P.L., 1988. [76] Caton, De agricultura, éd. W. D. Hooper et H. B. Asr, Londres/Cambridge (Mass.), Heinemann/Harvard Univ. Press, 1934.

[77] Caton, De agricultura, éd. Mazzarrno, Leipzig, Teubner, 2º edit. 1982.

[78 ] Caton, De l'agriculture, éd. R. Goujard, (C.U.F.), Paris, Les Belles Lettres, 1975.

[ 79$]$ Caron, Vom Landbau. Fragmente. Alle erhaltenen Schriften, lat. u. deutsch,

[80] P. THIeLscher, Des Marcus Cato Belehrung über die Landwirtschaft, Berlin, Duncker \& Humblot, 1963

[81] S. Boscherrn, Lingua e scienza greca nel De agricultura di Catone (Ricerche di storia della lingua latina, VIII), Rome, Ed. dell'Ateneo, 1970.

[ 82 ] L. CaLbou Monterusco, «Cato Agr. 74-121», GIF 32, 1980, 209-228.

[83] E. ŠImovičova, «Zur Frage der Arbeitorganisation und ihrer Terminologie in Catos Schrift De agriculturaw, GLO 15-16, 1983-84 [1987] 5-24. César

[ 84 ] B. F. Schemann, Caesars Wortschatz. Vollständiges Lexikon zu den Schriften Bellum Gallicum, Bellum Civile, Bellum Africanum, Bellum Alexandrinum

[ 85 ] C. M. Birch, Concordantia et index Caesaris, Hildesheim, Olms, 1988 (2 vols.).

[86] W. Wimme, Die technische Seite von Caesars Unternehmen gegen Avaricum (AAWN, 1973, 9), Wiesbaden, Steiner, 1974.

Varron [ 87 ] B. Carpauns, Stand und Aufgabe der Varroforschung (mit. einer Bibliographie
[ 88 ] W. W. BrigGs, Concordantia in Varronis libros De re rustica (with the techn. ssist. of T. R. Whrtre and C. G. Smrrezy), Hildesheim ,Olms, 1983.

[ 89 ] Varron, L'économie rurale. Livre I, éd. J. Heurgon, (C. U. F.), Paris, Les Belles Lettres, 1978.

[ 90 ] Varron, L'économie rurale. Livre II, éd. Ch. Gutraud, (C. U.F.), Paris, Les Belles Lettres, 1985.

[ 91 ] J. KoLENDo, «L'agriculture en Apulie d'après Varron, $R R$ 1,29,2», $D H A$, 1979, $267-271$.

[92] J. Coldart, «Varron et Pline l'Ancien. Remarques sur le style des deux auteurs techniques, Ktema $4,1979,161-168$

\section{Vitruve}

[ 93 ] L. Callebat, P. Bourer, Ph. Fleury, M. Zuinghedau, Vitruve. De architectura. Concordance, Hildesheim, Olms, 1984

[ 94 ] Vitruve, De architectura libri decem, éd. C. Fensterbusch, Darmstadt, Wiss. Buchgesellsch., 1964.

[ 95 ] VIrRuve, De l'architecture - Livre I, éd. Ph. Fleury, Paris, Les Belles Lettres, 1990.

[96] VItruve, De l'architecture-Livre VIII, éd. L CaLlebat, Paris, Les Belles Lettres, 1973.

[ 97 ] Virpuve, De l'architecture-Livre IX, éd. J. Soubiran, Paris, Les Belles Lettres, 1969.

[98] Virruve, De l'architecture-Lìre X, éd. L. CaLlebat et Pr. Fukury, Paris, Les Belles Lettres, 1986

[99] VIrruve, De l'architecture-Livre III, éd. P. Gros; Paris, Les Belles Lettres, 1990.

[100] Actes du colloque de Tours, «Les traités d'architecture de la Renaissance» (1-11.781), Paris, Picard, 1988

[101] Actes du colloque de Darmstadt, «Vitruv-Kolloquium des Deutschen Archäologen Verbandes» (17-18.6.82), (THD-Schriftenr. Wissensch. \& Technik, 22) (1984.

[102] Actes du colloque de Berlin, "Bauplanung und Bautheorie der Antike» (16-18.11.83) (Diskussionen zur archäol. Bauforschung. IV. Dt. Archäol. Inst.), Berlin, Wasmuth, 1984.

[103] Actes du colloque de Strasbourg, «Le dessin d'architecture dans les sociétés antiques (27.1.84), Strasbourg, 1985.

[104] Actes du colloque de Londres, "Vitruvius and his Influence» (15.12.86), (Warburg Institute Surveys and Texts), à paraître.

[105] Actes du colloque de Leyde, «Vitruvius' De Architectura and the Hellenistic and Republicain Archieclure (2023187), Leyde, 1989.

[106] H. KnвLL, Vitruvs Architekturtheorie, Versuch einer Interpretation, Darmtadt Wissensch. Buchgesellsch, 1985. 
[107] E. Romano, La capanna e il templo: Vitruvio o dell'architectura, Palerme, Palumbo, 1987

[108] J. voN FREBDEN, Oikia kyrrestou, Studien zum sogennanten Turm der Wind in Athen, Rome, Bretschneider, 1983.

[109] B. WEsENBERG, Beiträge zur Rekonstruktion griechischer Architektur nach literarischen Quelten, (MDAI. A, 9), Berlin, Gebr. Mann, 1983.

[110] E. Rawson, Intellectual Life in the Late Roman Republic, Londres, Duckworth, 1985.

[111] J. Adam, «Gromate et chorobate. Exercices de topographie antique», MEFR 1003-1029.

[112] E. GABBs, «La rifondazione di Salapia», Athenaeum 61, 1983, 514-516.

[113] C. Tosi, «La città antica e la religio nel De architectura di Vitruvio», CRDAC 2 [1980-81] 1984, 425-439.

[114] W. WEEER, «Vitruvius und das Drahsteil», AW 18, 4, 1987, 57.

[115] J. I. Porrer, «Putting things into perspective. Scaenographia and Vitruvian aesthetics (De Arch. 1,2,2 et 7, pr. 11)", résumé dans AAPhA, 1987, 61

[116] M. VIcrerss, «Persepolis, Vitruvius and the Erechtheum Caryatids. The Iconography of Medism and Servitude», RA, 1985, 3-28.

[117] G. Barbe Coquelin DE Liste, «L'Espagne de la Renaissance et Vitruve: Medidas del Romano de Diego de Sagredo», Caesarodunum 18 bis «Présence de l'archtecture et de l'urbanisme romains»), Paris, Les Belles Lettres, 1983, 159-16.

[118] P. Mylonas, «De architectura. Two Anniversaries of the Latin Text», Archaiologia $21,1986,68-82$.

[119] N. Paguara, "Vitruvio da testo a canone», Memoria dell'antico nell"arto italiana III. Dalla tradizione all' archeologia, Turin, 1986, 5-85,

[120] A. Novara, «Les raisons d'écrire de Vitruve ou la revanche de l'architecte", $B A G B, 1983,284-308$

[121] J. André, «La rhétorique dans les préfaces de Vitruve. Le statut culturel vol, 3, Univ, degli studi di Urbino, 1988, 265-289.

Virgile

[122] W. Sugrbaum, "Spezialbibliographie zu Vergils Georgica», ANRW II, 31(1980) 395-499.

[123] VIRGLE, Géorgiques, éd. E. DE SAINT-Denis, (C. U. F.), Paris, Les Belles Lettres, 1956.

[124] VIrGLLE, Georgiche, introd. di G. B. ConTe, testo, trad. e note a cura di A. Barghesi, Milan, Mondadori, 1980.

[125] VIrGILE, Georgica, hrsg., übers. \& komm. von M. ERREN, Heidelberg, Winter, 1985. [126] G. B. MrLes, Virgil's Georgics. A new interpretation, Berkeley/Los Angeles,
Univ. of California, 1980.

[127] A. SALVATORE, «Le api in Virgilio e in Varrone», Vichiana 6, 1977, 40-54.

[128] A. Cossarini, «Le Georgiche di Virgilio. Ideologia della proprietà», I: GFF 1, 1978, 83-93; II: GFF 2, 1979, 3-12

[129] R. MarTin, «Statut, fonction et sens du livre II des Géorgiques", VL 76, 1979, 2-15.

[130] G. KroMER, «The didactic tradition in Vergil's Georgics», Ramus 8, 1979, 721.

[131] J. A. Fernádezz Delgado, «Tradición hesiódica de las Geórgicas», Helmantica 82, 281-290.

[132] F. Murcke, «Life on Earth or the Man on the Land», Classicum 8 (Sidney 82, 17-22.

[133] A. GriLi, «Agricoltura e poesia nelle Georgiche», A\&R 28, 1983, 4-20.

[134] A. Marastons, «Virgilio nella letteratura agricola romana», Virgilio nostro antico. Atti delle celebrazioni per il bimillenario virgiliano in Calvisano,

[135] M. S. SpurR, «Agriculture and the Georgics», G\&R 33, 1986, 164-186.

[136] G. ForNI, «L'aratro a carrello in Virgilio", Atti del Convegno mondiale scien tifico di studi su Virgilio (Mantoue - Naples - Rome 19-24.9.81), Milan, Monda

[137] O. Pasquatemtr, «Il vocabolo ingenium in Virgilio», Euphrosyne 12, 1983-84,

\section{Columelle}

[138] R. MARTrN, «Etat présent des études sur Columelle», ANRW II, 32.3 (1985) $1959-1979$

[139] Berts G. G. / Ashwortr W. D., Index to the Uppsala Edition of Columella (Acto Universitatis Upsaliensis, 6), Uppsala, Almquist/Wiksell, 1971

[140] Cosumelze, Zwölf Bücher ïber die Landwirtschaft. Buch eines Unbekannten uber Baumzichttung, lat-dt., hrsg. \& übers. von W. RIcHTer (Namen-\& Wortregister von R. HEINE), Munich, Artemis-Verl., vol. 1, 1981; vol. 2, 1982.

[141] Columelle, De re rustica, livre X, éd. E. de SatnT-Denis, (C.U.F.), Paris, Les Belles Lettres, 1969.

[142] Columelle, Les arbres, éd. R. Goujard, (C. U. F.), Paris, Les Belles Lettres, 1986 [143] G. Maggiubli, «Il lessico non-virgiliano del X libro di Columella», Orpheus N.S. 1, 1980, 126-151.

[144] P. SÁEz Fernández, «Columela, RR XII, 52, 6. Canalis et solea», Habis 14, 1983, 147-152.

[145] P. P. CoRserri, «L'apport de la tradition indirecte à l'établissement du texte de Columelle, Res rustica, livre VI», Etudes de Lettres. Revue de la Faculte des Lettres de Lausanne, Janvier-Mars 1986 ("Sciences et techniques à Rome») pp. 33-44. 
Apicius

[146] Aprcius, De re coquinaria, éd. M. E. Milham, Leipzig, Teubner, 1969

[147] Aprcius, L'art culinaire, éd J. AndRé, (C.U.F.), Paris, Les Belles Lettres, 1974.

[148] R. MaIER, «Index instrumentorum coquinariorum apud Apicium atque Petronium inuentorum», VoxLat 23, 1987, 351-353.

[149] F. MARchese, «Aspetti della lingua tecnica di Apicio», AATC 38, 1987, 9-102 Frontin

[150] J. Costas Ropríguez, Frontini index, Hildesheim, Olms, 1985

[151] Frontrn, Les aqueducs de la ville de Rome, éd. P. Grimau, (C. U.F.), Paris, Les Belles Lettres, 1961.

[152] Frontin, De aquaeductu urbis Romae, éd. C. Kunderewicz, Leipzig, Teubner, 1973.

[153] Frontin, Los acueductos de Roma, ed. crítica y trad. T. González Rotan, Madrid, Consejo Superior de Investigaciones Científicas, 1985.

[154] Frontin, Kriegstisten, lat. und deutsch von G. Bendz, Berlin, Akademie-Verlag, $2^{\circ}$ éd. 1978 (1963).

[155] Frontin, De agri mensura, por P. Resina Sola, Granada Univ., 1984.

[156] Frontrs, The Stratagems and the Aqueducs of Rome, éd. C. E. Bennetr (Loeb Classical Library), Londres/Cambridge (Mass.), Harvard Univ./Heinemann, 1969

[157] F. Hernandez-GonzáLez, «Frontino y el léxico de las aguas», Tabona 4, 1983, 253-265.

Pline l' Ancien

[158] G. Seprat «Pline l'Ancien. Etat présent des études sur sa vie, son oeuvre et son influence», ANRW II, 32.4 (1986), 2069-2200.

[159] G. ForNI, «Il plaumaratum (aratro a carrello) di Plinio nel quadro della (toria del'aratrocultura in Italiaw, Tecnologia, economia e società nel mondo romano. Atti del Convegno di Como (27-29.9.79), 99-120.

[160] J. KorsNoo, «Origine et diffusion de l'araire à avant-train en Gaule et en Bretagnen, $C H$ 24, 1979, 61-73.

[161] H. LoeBER, «Bericht über alte Technologien der Glaserzeugung und Glasve arbeitung», $A \& N 1,1977,42-48$

Hygin

[162] Hygrn, Liber de metatione castrorum, éd. A. GriLtone, Leipzig, Teubner, 1977.

[163] Hygin, Des fortifications du camp, éd M. Lenorr, (C. U.F.), Paris, Les Belles Lettres, 1979.
[164] A. GriLlons, «Sul De metatione castrorum dello pseudo-Igino», Latomus 36,

[165] A. GriLone, «Note critiche al testo del De metatione castrorum dello pseudo-Igino», Studi italiani di filologia classica 49, 1977, 255-266.

[166] A. GriLLone, «Sul De metatione castrorum dello pseudo-Igino. Il linguaggio di un geometra del III secolon, Philologus 126, 1982, 247-264.

[167] E. BirLEy, «The dating and character of the tract De munitionibus castrorumn, Romanitas-Christanitas. Untersuchungen zur Geschichte und Literater der römischen Kaiserzeit. J. STRAUB zum 70. Geburtstag, Berlin, De Gruyter 1982, 277-281.

Cetius Faventius

[168] H. PLommer, Vitruvius and later roman building manuals, Cambridge, Univ. Press., 1973.

[169] P. PatTenden, «Sundials in Cetius Faventinus», CQ 29, 1979, 203-212. Ammien Marcellin

[170] J. M. ALonso-Nứ̃EZ, «Ammianus Marcellinus in der Forschung von 1970 bis 980», AAHG 36, 1983, 1-18

[171] М. Снтаво, Index uerborum Ammiani Marcellini, Hildesheim, Olms, 1983 [172] L. Viansino, Ammiani Marcellini rerum gestarum lexicon, Hildesheim, Olms, 1985.

[173] G. J. D. E. ARcrвoL, A Concordance to the Works of Ammianus Marcellinus (Phoenix sup. XIII), Toronto, Univ. Press, 1980 (microfiches).

[174] Ammien Marcedirin, Histoire. Livres XXIII-XXV, éd. J. Fontaine, (C.U.F.), Paris, Les Belles Lettres, 1977.

[175] N. J. E. Aussin, Ammianus on Warfare. An Investigation into Ammianus' Military Knowledge (Coll. Latomus 165), Bruxelles, Latomus, 1979. [176] M. F. A. Bror, «Bombast oder Kunstfertigkeit. Ammians Beschreibung der
Ballista $(23,4,1-3) »$, RhM 120, 3-4, 1977, 331-345.

De Rebus Bellicis

[177] De Rebus Bellicis, éd. R. I. IReLand, Leipzig, Teubner, 1984.

[178] S. ConDoRell, Riforme e tecnica nel De rebus bellicis, testo con comm. e vers.

[179] M. W. C. Hassar, De rebus bellicis (Part I: Aspects of the De rebus bellicis papers presented to Professor E. A. THompson. Part II: The text, edited, translated and presented, with Commentaries on Text, Language and Style, by R. I. InELAND) (British Archeological Reports, International Series 69), 
VARIA NOSCENDA

[180] H. BRANDr, Zeitkritik in der Spätantike. Untersuchungen zu den Reform vorschlägen des Anonymus De rebus bellicis, Munich, C. H. Beck, 1988.

[181] A. CERATI, «Pour la datation classique du De rebus bellicis», Etudes offertes à J. Macquerron, Aix en Provence, Fac. de Droit, 1970, 160-167.

[182] S. Mazzarino, «Il De rebus bellicis e la Gratiarum actio di Claudio Mamertinos, Studi FerRero, Turin, 1971, 209-214.

[183] B. BaLDwin, «The De rebus bellicis», Eirene 16, 1978, 23-29.

[184] S. Kors, «Eine moderne Imperialismus-Theorie im Anonymus De rebus bellicis?n, $\Phi$ i $\lambda$ la., X Xopiv. Miscellanea di studi

[185] S. Korв, «Finanzprobleme und soziale Konflikte aus der Sicht zweier spätantiker Autoren (Scriptores Augustae und Anonymus De rebus bellicis), hist. Abh. 28), Cologne, Böhlau, 1980.

[186] G. BoNaMENTE, "Considerazioni sul De rebus Bellicis», AFLM 14, 1981, 9-49.

[187] A. E. Astri, «Observations on the De rebus bellicis», Studies in Latin LiteA. A. Astiv, «Observations on the De rebus bellicis», Studies in Lates, Latomus, rature and
$1983,388-439$.

[188] D. ForaboschI, «Economia e guerra nel De rebus bellicis», Studi in mem. d C. CATTI, Milan, 1987, 111-127.

[189] I. G. Marrk, «The Giessen, Parma and Piacenza Codices of the Notitia Dignitatum with some related Texts», Latomus 27, 1968, 96 sqq

[190] I. G. Mares, «The Barberinus and Münich Codices of the Notitia dignitatum omnium», Latomus 28, 1969, 960 sqq.

[191] C. EGGER, «De nonnullis uerbis inferiore Romanorum aetate factis», Latinitas 18, 1970, 8-17

Végèce

[192] R. SabLayroules, «Bibliographie commentée de Végèce», Armée romaine et provinces, III, Paris, Presses

[193] V́́ćçce, Epitoma rei militaris, éd. C. Lang, Leipzig, Teubner, $2^{\circ}$ édit. 188 (1869), réimpr. 1967.

Palladius

[194] PALLADII RVTILII TAVRI AEMILIANI Vir Illustris Opus agriculturae. De ueterinaria medicina. De insitione, éd. R. H. RocGERs, Leipzig, Teubner, De ueterin
1975.

[195] Paldadius, Traité d'agriculture. Livres I et II, éd. R. Martin, (C.U.F.), Paris, Pelles Lettres, 1976.

[196] R. H. RodGers, An introduction to Palladius (BICS Suppl. 35), Londres, Univ., 1975.

[197] Cuc Jose AC 44, 1975, 2, 664-667.

[198] E. FrezouLs, "La vie rurale au Bas-Empire d'après l'oeuvre de Palladius», Ktema 5, 1980, 193-210

\section{ETUDES SUR LES TEXTES TECHNIQUES}

a. Études générales

[199] B. Deinlein, Das römische Sachbuch, Diss. Erlangen, 1975.

[200] B. Grut, Les mécaniciens grecs. La naissance de la technologie, Paris, Seuil,

[201] C. ре Mвo, Lingue tecniche del latino, Bologne, Patron, 1983.

[202] J. Dubors, «Structures lexicales et langues techniques» dans A. REY, La lexicologie, Paris, Klincksieck, 1970, 189-197 (= reprise de J. Dubors, «Les problèmes du vocabulaire technique», Cahiers de lexicologie 9, 1966, 2).

b. Etudes spécialisées dans un domaine

Architecture

[203] R. Ginouves et R. MARTin, Dictionnaire méthodique de l'architecture grecque an

[204] B. WrsENBERG, Beiträge zur Rekonstruktion griechischer Architektur nach

[205] B. WESENBERG, «Zu den Schriften der griechischen Architekten», Bauplanung und Bautheorie der Antike, Disk. zur archäolog. Bauforschung 4, 1983, 39 sqq. Agriculture

[206] M. G. BRuno, Il lessico agricolo latino, Amsterdam, Hakkert, 1969 .

[207] S. ANDREI, Aspects du vocabulaire agricole latin, Rome, L'«Erma» di Bretschneider, 1981.

[208] K. D. WHrre, «Roman Agricultural Writers I: Varro and his Predecessors ANRW I, 4 (1973) 440-497.

[209] R. MARrin, Recherches sur les agronomes latins et leurs conceptions économiques et sociales, Paris, Les Belles Lettres, 1971. Guerre

[210] E. L. WнEеLER, Stratagem and the Vocabulary of Military Trickery, (Suppl. Eremosyne), Leiden, Brill, 1988. 

[211] PH. FLEURY, «Vitruve et la nomenclature des machines de jet romaines»,
$R E L$ 59, 1981, 216-234.

[212] V. GuUfres, La letteratura de re militari. Appunti per una storia degli ordinamenti militari, Naples, Jovene, 1974

\section{ETTUDES SUR LE CONTENU TECHNIQUE}

Instruments bibliographiques

[213] R. J. ForBss, Bibliographia antica, Philosophia Naturalis X. Science and Technology, Leyde, 1950 (Suppl. II: 1950-1962, Leyde, 1963).

[214] F. Russo, Eléments de bibliographie de l'histoire des sciences et des techn ques, Paris, Hermann, $2^{\circ}$ édit. 1969 (1954).

[215] E. S. FerGuson, Bibliography of the History of Technology, Cambridge (Mass.) Inst, of Technology Press, 1968

[216] J. P. Ot.eson, Bronze Age, Greek and Roman Technology. A select annotated ry of Science and Technologie 13) Bew-York/Londres, Garland Publ, 1986.

a. Études générales

[217] History of Technology, sous la dir. de C. Singer, E. J. Holm yard, A. R. Hall, T. 1. WhuAars, II. The Medlterranean Civilizations and the Middle Ages, Oxford, Clarendon Press, $2^{\circ}$ édit. cor. 1957 (1956) (réimpr. 1962, 1967, 1972).

[218] Histoire des techniques, sous la dir. de B. Grute (Encycloplédie de la Pléiade), Paris, Gallimard, 1978.

[219] D. Hin, A History of Engineering in Classical and Medieval Times, Londres, Croom Helm, 1984.

[220] H. HodGes, Technology in the Ancient World, Londres, Allen Lane, 1970.

[221] R. J. For8ss, Studies in the Ancient Technology, Leiden, Brill, 1956-71 (9 vols.).

[222] J. G. Landels, Engineering in the Ancient World, Londres, 1978.

[223] Recherches sur les artes à Rome (Séminaire de $3^{\circ}$ cycle, Dijon, oct. 1978) (Publications de l'Université de Dijon 58), Paris, Les Belles Lettres, 1978.

[224] Scienza e tecnica nelle letterature classiche (Seste gionarte filologiche genovesi 23-24 feb. 1978) (Publ. de l'Inst. di Fil. Class. \& Med. 64), Gênes, Fac. des Lettres, 1980.

[225] L. A. \& J. A. Humer, The Roman Engineers, Cambridge Univ Press, 1981

[226] K. D. WHrre, Greek and Roman Technology, Londres, Thames and Hudson, K. D. [227] Le Bois dans la Gaute Romaine et les provinces voisines (Actes du colloque
1985. Caesarodunum 21), Paris, Ed. Errance, 1986.
2288 H. W. P P Talanta 5, 1973, 6.47.

[229] R. Haror, «La technique romaine», La Recherche 10, 1979, 364-372.

\section{b. Études spécialisées}

ARCHITECTURE ET CONSTRUCTION

229 bis] P. Gros, Aurea templa. Recherches sur l'architecture religieuse de Rome à l'époque d'Auguste (BEFAR 231), Paris, De Boccard, 1976.

[230] J. J. Couston, Greek Architects at Work. Problems of Structure and Design, Londres, 1977

231] J. P. Adar, La construction romaine, Matériaux et techniques, Paris, Picard, 1984

232] H. Laurer, Die Architektur des Hellenismus, Darmstadt, Wissensch. Buchgesellsch., 1986.

[233] A. Ghequiere, P. Overlau, G. Kelner, R. Caudano, J. Verbist, «Analyse du Pompéi par la méthode de la spectroscopie E. S. C. A.», $L E C 41,1973,402-418$.

[234] D. Cosmowrounss, «A propos d'un chapiteau de Délos. Le problème du tracé des volutes ioniques dans 1'Antiquité, Etudes Déliennes ( $B C H$ sup. I) Paris, De Boccard, 1973, 137-146.

[235] J. J. Courron, «Lifting in Early Greek Architecture», JHS 94, 1974, 1-19.

[236] V. Furban et P. Bisseger, «Les mortiers anciens. Histoire et essai d'analyse scientifique, Zeitschrift für Schweizerische Archä口logie und Kunstgeschichte $32,1975,166-178$.

[237] Dossiers de l'archéologie, 25, Nov-Déc. 1977 «Comment construisaient les Romains?».

[238] J. P. AdAm, «A propos du trilithon de Baalbek, le transport et la mise en oeuvre des mégalithes», Syria 54, 1977, 1-2, 31-63.

[239] A. GAudio, "Comment bâtissaient les Romains?», Arch. 114, 1978, 50-59.

[240] J. P. Adam et P. VarenNE, «Une peinture romaine représentant une scène de chantier», $R A, 1980,216-238$

AGRICULTURE

Généralités

[241] K. D. Wнrтe, A Bibliography of Roman Agricultule (Bibliogr. in Agricult. Hist. I), Univ. of Reading, 1970.

[242] K. D. WHтrs, Roman Farming New-York, Cornell, 1970

[243] K. D. Wнтте, Farm Equipment of the Roman World, Cambridge Univ. Press, 
[244] J. KoLENDo, L'agricoltura nell'Italia romana (Tecnice agrarie e progresso economico dalla repubblica al principato), (Biblioteca di storia antica 10) Rome, Riuniti, 1980.

[245] «La terre et les paysans dans l'Antiquité classique», Pallas 29, 1982

[246] O. WIIANDER, Exploitation of Water-Power or Technological Stagnation? A Reapraisal of the Productive Forces in the Roman Empire, Lund, Gleerup, Moulin

[247] R. Freneaux, «Barbegal», Caesarodunum 5, 1970, 309-313,

[248] N. G. CALvERT, «The Mono-Kairos Windmills of Lasithi», ABSA 70, 1975, 51-57.

[249] N. CorReia Borges, «Mós manuais de Conimbriga», Conimbriga 17, 1978, 113-132.

[250] O. WIKANDER, «Water-mills in Ancient Rome», Opuscula Romana 12, 1979, 13-35,

[251] P. Roos, «For the fiftieth Anniversary of the Excavation of the Water-mill
at Barbegal: A Correction of a Long Lived Mistakes, RA 2, 1986, 327-334. ] R. J. SpaIN, «The Roman Water-mill in the Athenian Agora. A new View of Evidence», Hesperia 56, 1987, 335-353.

[253] D. P. S. Рвасоск, «The Mills of Pompei», Antiquity 63, 1989, 205-214. Pressoir

[254] A. NEYSEs, «Drei neuentdecke Gallo-römische Weinkelterhauser im Moselgebiet», AW 10, 2, 1979, 56-59.

[255] P. Brungau et P. Fraisse, «Un pressoir à vin à Délos», $B C H$ 105, 1981, 127-153.

[256] J. KoLENDo, «Origine et diffusion de l'araire à avant-train en Gaule et en Herse

[257] J. KoLENDo, «Avènement et propagation de la herse en Italie antique», Archeologia 22, 1971, 104-120.

Tudicula

[258] J. P. LAPorTs, «La tudicula, machine antique à écraser des olives, et les massues de bronze d'Afrique du Nord", résumé dans BCTH X, B, 1974, 167-174. GUERRE

\section{Généralités}

[259] E. W. Marsden, Greek and Roman Artillery. Historical Development, Oxford, Wress, 1969.

[260] G. WEBsTer, The Roman Imperial Army, Londres, Adam \& Charles Black,

[261] Y. Garlan, La guerre dans l'Antiquité, Paris, Nathan, 1972

[262] J. Harmand, La guerre antique, de Sumer à Rome, Paris, P.U.F., 1973.
[263] O. LendLE, Schildkröten. Antike Kriegsmaschinen in poliorketischen Texten (Palingenesia 10), Wiesbaden, Steiner, 1975.

[264] O. LENDLE, Texte und Untersuchungen zum technischen Bereich der antiken Poliorketik (Palingenesia 19), Wiesbaden, Steiner, 1983.

[265] G. BRIzzI, Studi militari romani (Studi di Storia Antica 8), Bologne, CLUEB, 1983.

[266] Y. Le Bонвс, L'armée romaine du Haut-empire, Paris, Picard, 1989.

\section{Machines de jet}

[267] D. BAATz, «Das Torsiongeschütz von Hatra», AW 9, 1978, 4, 50-57.

[268] D. BAATz, «Recent Finds of Ancient Artillery», Britannia 9, 1978, 1-17.

[269] D. BAATZ, «Teile hellenistischer Geschütze aus Griechenland», $A A$, 1979, 68-75.

[270] D. BaAtz, «Ein Katapult der Legio IV Macedonica aus Cremona», $M D A I(R)$ D. BaArz, «Ein Kat $1980,283-299$.

[271] D. BaAtz, «Eléments d'une catapulte romaine trouvée à Lyon», Gallia 39, $1981,201-209$

[272] D. BAATz, «Hellenistische Katapulte aus Ephyra (Epirus)», MDAI (A) 97, 1982,

\section{Machines diverses}

273] L. Poznanski, «Encore le coruus de la terre à la mer», Latomus 38, 1979, $652-661$.

[274] O. LendLe, «Antike Kriegsmaschinen», Gymnasium 88, 1981, 330-356. [275] M. A. Tomer, «La tecnica nel tardo impero romano; le macchine de guerra»,

\section{Adduction d'eau et machines pour extraire l'eau}

[276] T. SchroLre, Roman and Islamic Water-lifting Wheels, transl. by P. M. KatBope (Acta hist. scient. natural. et medic. 28), Odense, Univ. Press, 1973.

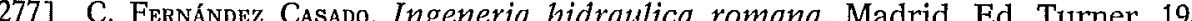

[278] Wasserversorgung im antiken Rom, Herausgeber: Frosirinvs-Gesellschaft Oldenbourg, $2^{\mathrm{c}}$ édit. 1983.

[279] J. Bonsis, L'eau dans l'Antiquité. L'hydraulique avant notre ère, Paris, Eyrolles, 1984

[280] J. P. Oteson, Greek and Roman Mechanical Water-lifting Devices: The History J. P. Oresson, Greek and Romann Mechanical Water-lifting Devices:
of Technology (Phoenix, Suppl. 16), Univ. of Toronto Press, 1984.

[281] L'homme et l'eau en méditerranée et au Proche-Orient, IV. L'eau dans l'agriculture. Seminaire de recherche $1982-83$ et journees des 22 et 23.10.83, sous la dir. de P. Louis, F. et J. Métral (Travaux de la Maison de l'Orient 14, 
[282] Ph. Leveau et J. L. Pallder, L'alimentation en eau de Caesarea de Maurétanie et l'aqueduc de Cherchel, Paris, 1976.

[283] W. Preprers, «Ein Rückschlagventil römischer Zeit im Rheinischen Landmuseum Bonn», $A W$ X, 1, 1979, 58-59.

[284] O. WIKANDER, "The Use of Water-power in Classical Antiquity», ORom 13, 1981, 91-104.

[285] T. HoDGE, "Les siphons inversés des aqueducs romains", Pour la Science, Août, 1985, 16-23.

A N N E X E

CLASSEMENT THEMATIQUE DES TEXTES TECHNIQUES Agriculture:

G: Hésiode
L: Caton

Varron

Virgile

Palladius

Construction:

L: Vitruve

Cetius Faventinus

Levage et traction des charges

G: Pseudo-Aristote

L: $\quad$ Vitruve

Adduction d'eau et machines

pour extraire l'eau:

G: Philon
Héron

L: Vitruve

Cuisine:

L: Apicius
Guerre:

G: Ené

Biton

Philonénée

Héron

Apollodore Polyen

+ Tacticiens

L: César

Vitruve

Frontin

Hygin

Ammien Marcellin

De rebus bellicis

Modestus

Mesures et instruments de mesure:

G: Philon

$\begin{array}{ll}\text { G: } & \text { Héron } \\ \text { L: Vitruve }\end{array}$

Vitruve
$\quad+$ Gromatici

Thaumaturgie:

G: Philon

L: Héron

+ Varron (La volière)

Centre d'Etudes et de Recherches pour l'Antiquité,

Université de Caen 